\title{
Multicriteria assessment of advanced treatment technologies for micropollutants removal at large-scale applications
}

\author{
X.T. Bui ${ }^{a}$, T.P.T. Vo ${ }^{b}$, H.H. Ngo ${ }^{a, b, *}$, W.S. Guo ${ }^{b}$, T.T. Nguyen ${ }^{a}$ \\ ${ }^{\text {a }}$ Environmental Engineering and Management Research Group, Faculty of Environment and Labour Safety, Ton Duc Thang \\ University, Ho Chi Minh City, Viet Nam \\ ${ }^{\mathrm{b}}$ Centre for Technology in Water and Wastewater, School of Civil and Environmental Engineering, University of Technology \\ Sydney, Broadway, NSW 2007, Australia
}

* Corresponding author at: Environmental Engineering and Management Research Group, Faculty of Environment and Labour Safety, Ton Duc Thang University, Ho Chi Minh City, Viet Nam. E-mail addresses: h.ngo@uts.edu.au,ngohuuhao@tdt.edu.vn(H.H. Ngo)

\begin{abstract}
With the introduction and discharge of thousands of new micropollutants (MPs) every year, traditional water and wastewater treatment plants may be incapable of tackling them all. With their low concentrations and diver- sity in nature, MP removal encounters numerous challenges. Although some MPs are effectively eliminated via conventional treatment methods, most of them can easily escape and are retained in the discharged effluent. Therefore, advanced methods such as (i) adsorption, (ii) oxidation and advanced oxidation processes (O3 and O3-based advanced oxidation processes, UV/H2O2), (iii) membrane processes, and (iv) membrane bioreactors, become an inevitable approach. Despite the unsurprisingly vast number of papers on MP treatment available at present, most of these studies were carried out at a laboratory scale while only a few pilot- and full-scale stud- ies have experimented. Nevertheless, an indepth assessment of real-world MP treatment methods is extremely crucial for practitioners. To date, no paper has been dedicated to look at this issue. Therefore, this paper aims to review these large-scale treatment methods. First, the paper goes through the regulations and standards which deal with MPs in water courses. It will then assess these methods in various case-studies with reference to differ- ent criteria towards serving as a reference for further practical applications.
\end{abstract}

Keywords: Multicriteria assessment; Micropollutants; Advanced treatment methods

Abbreviations: AC, activated carbon; AOP, advanced oxidation process; BOD, biological oxygen demand; COD, chemical oxygen demand; DEET, N,N-Diethyl-metatoluamide; DEHP, diethylhexyl phthalate; DOC, dissolved organic carbon; EBCT, empty bed contact time; EDCs, endocrine disrupting compounds; GAC, granular activated carbon; HRT, hydraulic retention time; MBR, membrane bioreactor; MCPA, 2-methyl-4-chlorophenoxyacetic acid; MF, microfiltration; MLD, megalitres per day; MPs, micropollutants; MW, molecular weight; NP, nonylphenol; NPE, nonylphenol diethoxylate; NDMA, N-nitrosodimethylamine; NF, nanofiltration; NP, nonylphenol; PAC, powdered activated carbon; PCPs, personal care products; PPCPs, pharmaceuticals and personal care products; RO, reserve osmosis; SBR, sequence batch reactor; SRT, sludge retention time; SS, suspended solids; UF, ultrafiltration; UV, ultra violet; WFD, Water Framework Directive; WHO, World Health Organisation; WWTPs, wastewater treatment plants. 



\section{Contents}

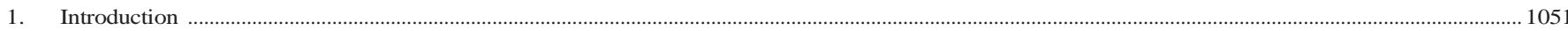

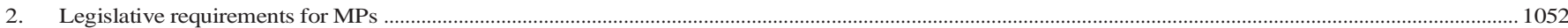

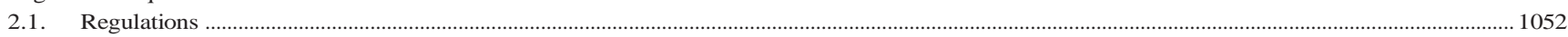

2.2. Case study - Swiss Government's National Research Program 50 ....................................................................................................................... 1052

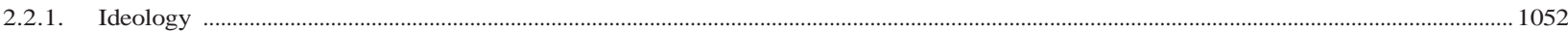

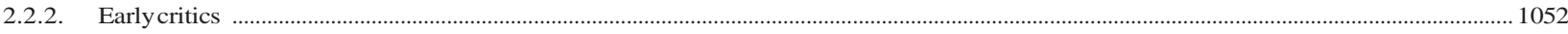

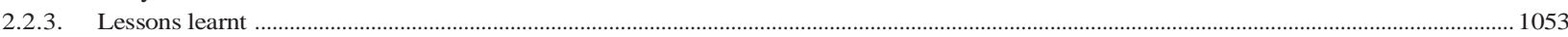

3. Multicriteria assessment of advanced treatment technologies ………………………………………………………………………………………………... 1053

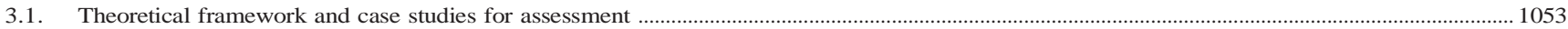

3.2. Range of treated pollutants, treatment efficiency and removal mechanisms ………........................................................................................................ 1053

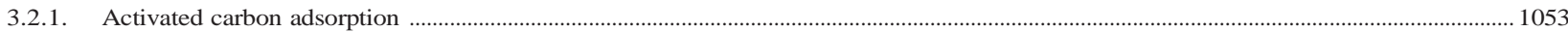

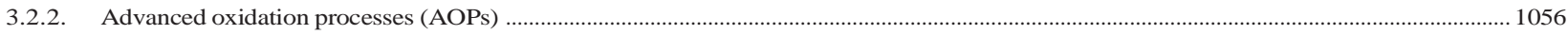

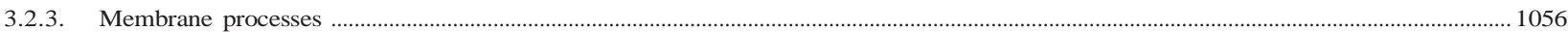

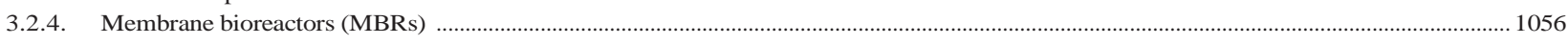

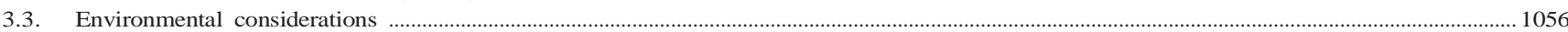

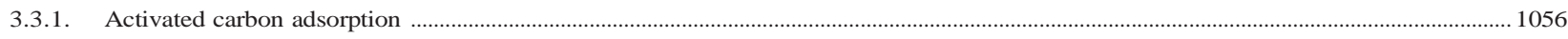

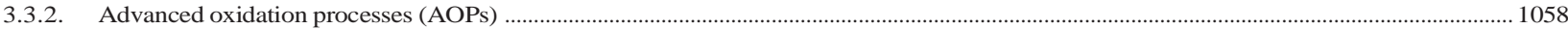

3.3.3. Membrane processes ............................................................................................................................................................................ 1061

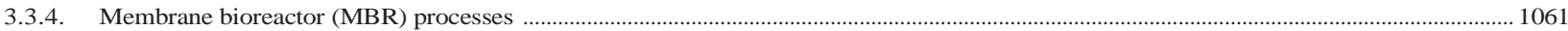

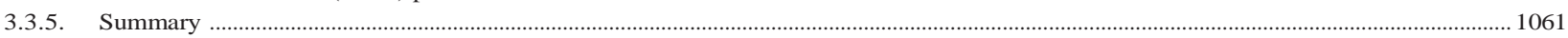

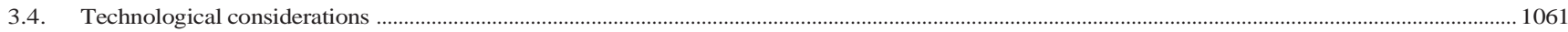

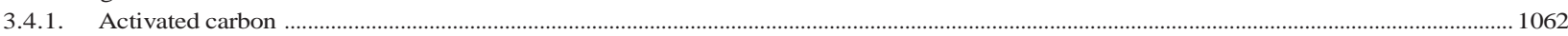

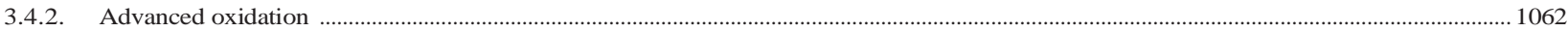

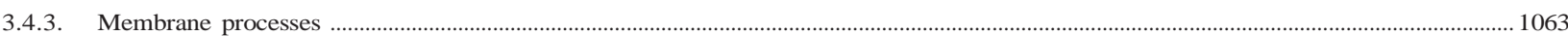

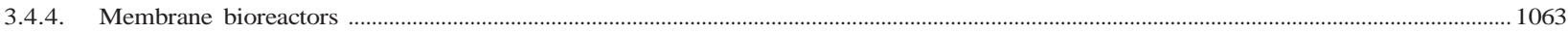

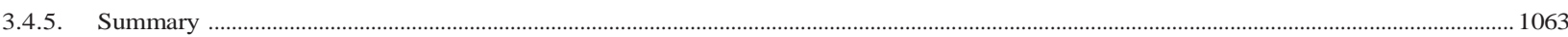

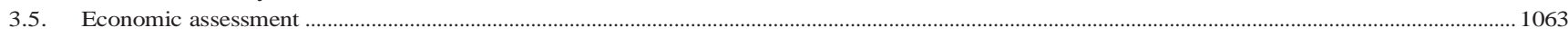

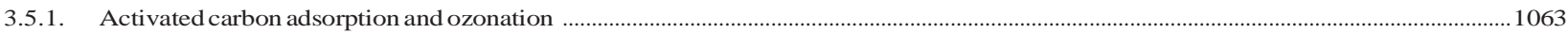

3.5.2. Comparison with other treatment processes ..................................................................................................................................................... 1064

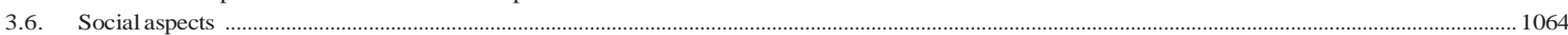

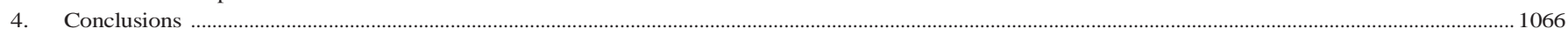

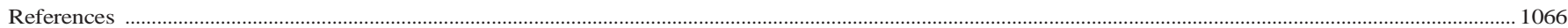

\section{Introduction}

To date, conventional water and wastewater treatment plants have successfully controlled basic pollutants such as organic matters, nutrients and pathogens. However, with the introduction and, consequently, discharge of thousands of new anthropogenic substances every year, traditional plants may fail to tackle them. A majority of these substances are called micropollutants (MPs) because they are present in the water environment in small amounts but can be highly toxic, even at extremely low concentrations (below several $\mu \mathrm{g} / \mathrm{L}$ ) (Luo et al., 2014). Basically MPs can be classified into four major groups, although there may be some overlaps between different groups, namely (i) metals, metalloids and radioactive elements (i.e., antimony, arsenic, cadmium, lead, mercury, radon, uranium); (ii) organic MPs (i.e., hydrocarbons, solvents, surfactants, personal care products, pesticides); (iii) hormones; and (iv) pharmaceutical products and endocrine disruptors (i.e., analgesics, antibiotics, antidepressants, betablockers, hypolipidemic, bronchodilators, and chemotherapy products) (Degremont, 2013).

These substances have not been encountered in any design process of traditional water and wastewater treatment plants. As Blackbeard et al. (2016) observed, there were 91 MP compounds consistently presented in the secondary-treated effluent from Eastern Treatment Plant (Australia). In another survey carried out by Thomaidi et al. (2015) in Greece, about 207 organic MPs were detected in different WWTPs, ranging from $1 \mathrm{ng} / \mathrm{L}$ to thousands $\mathrm{ng} / \mathrm{L}$. Components with highest concentrations for specific groups were acesulfame $(27,200 \mathrm{ng} / \mathrm{L}$, in artificial sweeteners group), nonylphenol diethoxylate (NPE) $(17,400 \mathrm{ng} / \mathrm{L}$, in endocrine disrupting compounds (EDCs) group), valproic acid (17,292 ng/ $\mathrm{L}$ in pharmaceuticals group), decamethylcyclopentasilane (6020 ng/L, in siloxanes group), tolytriazole (5773 ng/L, in benzotriazoles group), and perfluoroundecanoic acid (1281 ng/L, in perfluorinated compounds group) (Thomaidi et al., 2015). The Swiss National Research Program 50 investigated over a wide range of MP discharge sources and concluded that WWTPs were the key route for introduction of MPs and EDCs into the aquatic environment (Eggen et al., 2014).

Although effects of all MPs on the environment and human health are not fully understood, abundant scientific evidence has confirmed that some pharmaceuticals and EDCs (nonylphenol, NPE, nonylphenol monoethoxylate and triclosan) were a hazard to aquatic organisms (Logar et al., 2014; Jones et al., 2007) where they contributed to 99\% of toxicity to fish and Daphnia magna, and $98 \%$ to algae (Thomaidi et al., 2015). Even with the high dilution factor of rivers (N2000 times), MPs remained a risk for aquatic ecology with risk quotient values of 40-120 for algae, daphnia and fish (Thomaidi et al., 2015).

With MPs' low concentrations and diversity in nature, there are numerous challenges involved in their removal. Although some MPs are partly removed via conventional treatment methods through vaporisation or sorption onto activated sludge such as fragrances (40\%), nonylphenol monoethoxylate (13\%) and Bisphenol A (40\%) (Luo et al., 2014), most of them can easily escape from bioreactors and are retained in discharged effluents (X. Li et al., 2015). Therefore, advanced methods become an inevitable approach. Indeed, tertiary 
methods such as (i) adsorption, (ii) oxidation and advanced oxidation processes $\left(\mathrm{O}_{3}, \mathrm{O}_{3}\right.$-based advanced oxidation processes, $\left.\mathrm{UV} / \mathrm{H}_{2} \mathrm{O}_{2}\right)$, (iii) membrane processes, (iv) membrane bioreactors and their hybrid processes were studied extensively to remove MPs in the past decades.

Despite the number of papers on MPs treatment is unsurprisingly tremendous, most of them were experimented at a laboratory scale while only a few pilot- and full-scale studies have carried out. Nevertheless, an in-depth assessment of real-world MP treatments is extremely crucial for practitioners. As lab-scale experimental conditions are significantly different with actual water/wastewater matrix and operational conditions, a successful laboratory treatment process does not guarantee its success on a larger scale. Pilot-scale studies are, thus, required to simulate real treatment processes prior to full-scale execution. A proper pilot-scale plant with a whole set of "real" operational parameters can provide an accurate portrait of a full-scale plant, albeit some cautions of the scaling must be taken into account. Pilot-scale is often sufficient enough to extend our knowledge towards MP treatment of a particular treatment train. Nonetheless, learning from full-scale case studies is still the best option as it involves various aspects of a treatment process rather than efficiency alone.

To date, no paper has been dedicated to look at this issue. Therefore, this paper aims to review the treatment methods in pilot- and full-scale. First, it goes through the MP regulations in different countries and visits a case study of Swiss national strategy in their efforts to control MPs. It will then assess these large-scale methods in various case-studies with reference to different criteria towards serving as a reference for further practical applications.

\section{Legislative requirements for MPs}

\subsection{Regulations}

The fundamental question of how many or to what extent MPs should be regulated is very troublesome for any decision maker. This is because MP compounds are diverse in nature, even within the same family. To regulate a substance, an extensive research program must be done to provide a scientific background, including identification its source, fate, transformation and toxicity in different environments. To date, nearly 600 compounds have been defined but only a small number of them are regulated (Table 2). The fate of thousands of other MPs are unclear.

The Water Framework Directive WFD (2000/60/EC) was recognised as the first regional effort to address 33 priority substances. This directive has subsequently been refined by the Decision No. 2455/2001/EC and the Environmental Quality Standards Directive (2008/105/EC). In 2013, 12 more substances were added to the list, making up to 45 regulated compounds. However, there are many more problematic compounds present, apart from those compounds (Metz and Ingold, 2014).

At national level, Switzerland is the first country in the world imposing MP control at the point source. The proposal of Federal Office for the Environment to reduce MP loadings by $80 \%$ at selected WWTP outlets was approved by the Swiss parliament in 2011. Subsequently, the MP control in WWTP effluents was regulated in the Ordinance on Water Protection after a series of public consultation from 2012 to 2014, which will be in effect in 2016 (Audenaert et al., 2014). In the cases USA, there is no official control in MP elimination, except few states, i.e. California, issued their own regulations separately (Audenaert et al., 2014).

Whereas the release of some groups (i.e., pesticides and metals) declines due to stricter source reduction strategies, pharmaceutical discharge tends to increase over time (Metz and Ingold, 2014). The concerns over potential risks of some substances (especially antibiotics and EDCs) on water organisms and public health are ongoing. Nonetheless, it is unlikely that pharmaceutical compounds are regulated soon, although there was a proposal to add $17 \alpha$-ethinylestradiol, $17 \beta$-estradiol and diclofenac to the WFD list in 2011 (Johnson and Sumpter, 2015).
This is due to (i) their benefits and widespread uses for human health, (ii) inadequacy of scientific evidence on the impacts of these compounds in water courses, and (iii) lacking of systematic monitoring of various pharmaceuticals (NRMMC, 2015). One exceptional endeavour was the Australian government's effort in establishing threshold values for MPs, including pharmaceuticals, in secondary effluents for reuse in water supply areas in Australian Guidelines for Water Recycling: Augmentation of Drinking Water Supplies (NRMMC, 2008). In short, the comparison of control regulations in EU, USA, Australia and Switzerland was briefly presented in Table 1 . The preferred technologies were statistically summarised, rather than officially defined in any legal documents.

Table 2 presents several legislative requirements of MPs control in the water sector. The paper will focus on organic MPs, pharmaceuticals and steroid hormones - either regulated or non-regulated. This table presented different regulations for various types of MPs in a wide range of water environment. It can be seen that the concerns relating to MPs were bound strongly with drinking water rather than effluent discharge. To date, only Australian guidelines for water recycling: augmentation of drinking water supplies (NRMMC, 2008) and Report on public health criteria for direct potable reuse systems (Audenaert et al., 2014) have mentioned about standards for effluent discharge. It is noteworthy to mention that in Table 2, some MPs may fall into different classifications, depending on their nature and use.

\subsection{Case study - Swiss Government's National Research Program 50}

\subsubsection{Ideology}

Switzerland is the pioneer country to enforce legal obligations for minimization of MP loadings from municipal WWTPs. Four criteria were set up to screen whether a WWTP should be upgraded to control MPs discharge, based on the following principles (Eggen et al., 2014):

- Serving capacity of WWTP (N80.000 persons);

- Sensitivity of receiving waters (drinking water reservoirs or sensitive areas);

- Dilution capacity of receiving waters (N $10 \%$ of the dry-season stream flow);

Accordingly, 123 out of 750 WWTPs need to be improved, with the estimated costs of CHF 133 million (equivalent to 135 million USD) per year for the program lifespan of 24 years, from 2016 to 2040 (Logar et al., 2014). By imposing 80\% MP elimination target for the screened WWTPs, the national MP loading is expected to reduce by 50\% (Mulder et al., 2015).

With reference to the "polluter-pays principle", the financial contribution to the project is divided as following - 75\% from the central government and 25\% from the municipalities (Audenaert et al., 2014). Indicator compounds selected for monitoring the treatment processes are benzotriazole, carbamazepine, diclofenac, sulfamethoxazole and mecoprop. These MPs were selected because they often (i) appear in wastewater and surface waters, (ii) have a low removal rate in conventional WWTPs, (iii) represent a wide range of components in wastewater (pesticides, pharmaceuticals and industrial compounds) and (iv) can be detected easily by high-performance liquid chromatography (Eggen et al., 2014; Mulder et al., 2015).

\subsubsection{Early critics}

However, the Swiss proactive initiative was initially criticised being a premature decision for (i) not considering all ecological aspects, (ii) exposing a financial burden to the society, and (iii) providing ambiguous environmental benefits (Johnson and Sumpter, 2015; Jones et al., 2007). In contrast, Stamm et al. (2015) countered these critiques, considering that the decision was made with adequate scientific, economic and social data. From a technical viewpoint, several pilot- and full-scale projects have been tested for MP removal efficiencies, energy demands 
Table 1

Comparison of the status of emerging MP regulation in EU, USA, Australia and Switzerland (modified from (Audenaert et al., 2014)).

\begin{tabular}{|c|c|c|c|c|}
\hline & EU & Switzerland & USA & Australia \\
\hline Regulations & $\begin{array}{l}\text { - In development. } \\
\text { - Some WWTPs take proactive measures, espe- } \\
\text { cially in Germany. }\end{array}$ & - In place & $\begin{array}{l}\text { - In place for water reuse } \\
\text { schemes } \\
\text { - Some WWTPs take pro- } \\
\text { active measures }\end{array}$ & $\begin{array}{l}\text { - In place for water reuse schemes } \\
\text { - Some WWTPs take proactive measures }\end{array}$ \\
\hline $\begin{array}{l}\text { Indicator } \\
\text { compounds }\end{array}$ & $\begin{array}{l}\text { - In development, } \\
\text { - sometimes locally } \\
\text { - defined (e.g. France) }\end{array}$ & - Defined & - Defined for water reuse & $\begin{array}{l}\text { - Defined for water reuse, especially for } \\
\text { pharmaceuticals }\end{array}$ \\
\hline $\begin{array}{l}\text { Online } \\
\text { monitoring }\end{array}$ & - In development & - In development & - In development & - In development \\
\hline Criteria & $\begin{array}{l}\text { - Based on concentration (environmental qual- } \\
\text { ity standards), defined for surface waters }\end{array}$ & $\begin{array}{l}\text { - Based on removal (\%), de- } \\
\text { fined for WWTP effluents }\end{array}$ & $\begin{array}{l}\text { - Based on removal (log), } \\
\text { defined for water reuse }\end{array}$ & $\begin{array}{l}\text { - Based on concentration (environmental } \\
\text { quality standards), defined for water reuse }\end{array}$ \\
\hline $\begin{array}{l}\text { Preferred } \\
\text { technologies }\end{array}$ & $\cdot \mathrm{O}_{3}$ and/or PAC/GAC & $\cdot \mathrm{O}_{3}$ and/or $\mathrm{PAC} / \mathrm{GAC}$ & $\begin{array}{l}\text { UF/RO + oxidation } \\
\text { (water reuse) }\end{array}$ & $\begin{array}{l}\text { - Unclear trend, varied among } \mathrm{O}_{3}, \mathrm{PAC} / \mathrm{GAC} \text {, } \\
\mathrm{RO} \text { and MBR (water reuse) }\end{array}$ \\
\hline
\end{tabular}

and additional budget for upgrading and operation (Hollender et al., 2009). Full-scale upgraded plant's operational cost increases from $5 \%$ to $35 \%$, depending on the nature of current treatment technologies and upgrading options (Logar et al., 2014). In addition, the widespread and consistent upgrading of WWTPs definitely reduces the toxicity from MPs in the environment, and positively contributes to natural restoration and water reservoirs. Actually, a societal decision-making always involves a certain degree of uncertainty. Therefore, uncertainty is not an excuse for "non-action" scenario. The movement in Switzerland is also an opportunity to observe the responses of natural ecosystems from large-scale implementation of mitigation measures (Stamm et al., 2015).

\subsubsection{Lessons learnt}

Several lessons can be drawn from the case study of Swiss WWTP upgrading program for MP control. Firstly, a successful control of MPs must come from the government level with clearly defined goals

which are translated to legal obligations and practical implementation measures. However, it is not the only factor. The success to integrate MP control regulation into law was a result of a harmonious combination of top-down and bottom-up approaches. This decision-making process involved all relevant stakeholders from public, scientists and practitioners to politicians. They were consulted in a democratic manner over a long period of time with a variety of venues and methods. The key to its triumph was to raise public awareness on the effects of MPs and the MP control initiative. As a result, even this large-scale implementation has not proved its environmental effects yet (which often requires long-term evidences), it already brings higher perceptions in the public and politicians towards environmental issues. In addition, this case study provided a solid background for answering the question whether a WWTP should be upgraded and which MPs should be used as proxy. Although different countries may have different set of conditions, these basic properties such as (i) capacity of WWTPs and (ii) sensitivity and dilution factors of receiving waters must be taken into account.

\section{Multicriteria assessment of advanced treatment technologies}

\subsection{Theoretical framework and case studies for assessment}

Eggen et al. (2014) proposed several criteria to assess MP removal methods, such as (i) having a sound scientific and technical basis, (ii) based on broad societal and political acceptance, (iii) technically feasible, (iv) manageable, (v) pragmatic, (vi) adaptable in time and (vii) financially feasible. These criteria have been reorganised to evaluate MP advanced treatment alternatives, including (i) range of treated pollutants, treatment efficiency and removal mechanisms, (ii) environmental friendliness, (iii) simplicity of operation and maintenance, (iv) cost-effectiveness and (v) social acceptance.
The pilot- and full-scale case studies used in this paper are summarised in Table 3. Seven full-scale plants, either drinking water or wastewater treatment, and 14 pilot-scale cases within a decade have been assessed towards five above-mentioned criteria. The results from these cases will be discussed in the following sections.

\subsection{Range of treated pollutants, treatment efficiency and removal} mechanisms

It is noteworthy that concentrations of MPs vary greatly from one source to another. Pharmaceuticals, personal care products, EDSs and surfactants from raw municipal wastewater are often found in the range of $10^{-4}-10^{4} \mu \mathrm{g} / \mathrm{L}$ (Luo et al., 2014). Meanwhile, MPs in surface water could be pesticides, PPCPs, EDSs, surfactants and industrial substances resulting from agricultural run-off, municipal and industrial wastewater discharges. Surprisingly, some MPs presented in surface water with a higher concentration than in municipal wastewater, for example tetracycline $\left(10^{-4}-10^{3} \mu \mathrm{g} / \mathrm{L}\right.$ in surface water) (Cazes et al., 2014).

The removal of these contaminants is mainly based on their distinctive physio-chemical behaviours in certain conditions, including (i) hydrophobicity, (ii) absorbability, (iii) volatility, (iv) biodegradability, (v) charge, and (vi) molecular weight and size. As a rule of thumb, a group of substances with a similar characteristic will behave consistently towards a single treatment method. Table 4 simplifies the selection of suitable methods towards specific groups of substances on the ground of their physiochemical characteristics. Of course, due to the diversity of substance properties, prediction solely based on these characteristics may lead to conflicting results. Therefore, this table is not a comprehensive guideline, rather it aims to provide a better understanding on behaviour of compounds with comparable properties.

In the matrix of treatment process and physiochemical properties, MPs are classified as highly, moderately or insignificantly removed (Table 4). Obviously, one method cannot remove all contaminants. In some instances, an MP cannot be treated in one treatment can be removed by another method. For example, carbamazepine is hardly eliminated in any treatment other than advanced oxidation. The most challenging groups of MPs are those having (1) high water solubility, (2) low sorption coefficients, (3) low biodegradability and (4) high chemical stability (Zwiener, 2006).

\subsubsection{Activated carbon adsorption}

Activated carbon adsorption is a common method to remove odour, taste and organic compounds in water treatment plants, and currently being extended to remove MPs in WWTPs. Whereas granular activated carbon (GAC) is often utilised as a filtration medium, powdered activated carbon (PAC) is added in activated sludge or coagulation tanks (Luo et al., 2014). The adsorption processes are controlled by contact time (PAC) and empty bed contact time EBCT (GAC). This method is suitable for wastewater with $\mathrm{DOC}=10-20 \mathrm{mg} / \mathrm{L}$ because higher organic 
Table 2

Summary of common regulated and non-regulated MPs in water sector.

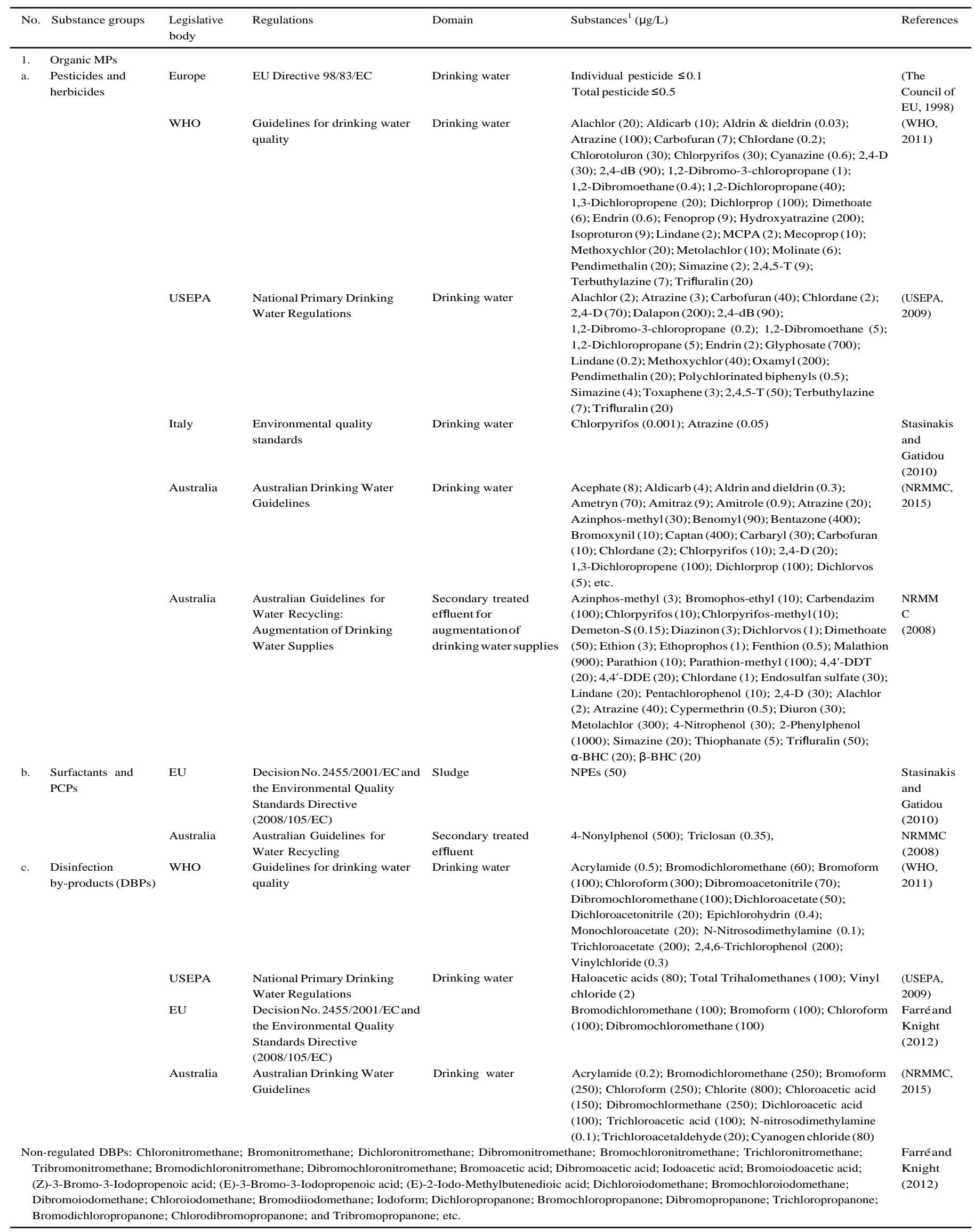


Table 2 (continued)

\begin{tabular}{|c|c|c|c|c|c|c|}
\hline No. & Substance groups & $\begin{array}{l}\text { Legislative } \\
\text { body }\end{array}$ & Regulations & Domain & Substances $^{1}(\mu \mathrm{g} / \mathrm{L})$ & References \\
\hline \multirow[t]{4}{*}{ d. } & \multirow[t]{4}{*}{$\begin{array}{l}\text { Solvents and other } \\
\text { organic compounds }\end{array}$} & WHO & $\begin{array}{l}\text { Guidelines for drinking water } \\
\text { quality }\end{array}$ & Drinking water & $\begin{array}{l}\text { Benzene(10); Carbon tetrachloride(4); 1,2-Dichlorobenzene } \\
\text { (1000); 1,4-Dichlorobenzene (300); 1,2-Dichloroethane } \\
\text { (30); 1,2-Dichloroethene (50); Dichloromethane (20); } \\
\text { Di(2-ethylhexyl)phthalate (8); 1,4-Dioxane (50); Edetic acid } \\
\text { (600); Ethylbenzene (300); Hexachlorobutadiene (0.6); } \\
\text { Nitrilotriacetic acid(200); Pentachlorophenol(9); Styrene } \\
\text { (20); Tetrachloroethene(40); Toluene(700); Trichloroethene } \\
\text { (20); Xylenes (500) }\end{array}$ & $\begin{array}{l}\text { (WHO, } \\
\text { 2011) }\end{array}$ \\
\hline & & USEPA & $\begin{array}{l}\text { National Primary Drinking } \\
\text { Water Regulations }\end{array}$ & Drinking water & $\begin{array}{l}\text { Benzene (5); Benzo(a)pyrene(PAHs) (0.2); Carbon } \\
\text { tetrachloride (3); 1,2-Dichlorobenzene (600); } \\
\text { 1,4-Dichlorobenzene(75); 1,2-Dichloroethane(5); } \\
\text { 1,2-Dichloroethene (7); Dichloromethane (5); } \\
\text { Di(2-ethylhexyl)phthalate (6); Dioxin (0.00003); } \\
\text { Ethylbenzene (700); Ethylene dibromide (0.5); Heptachlor } \\
\text { (0.4); Heptachlor epoxide (0.2); Hexachlorobutadiene (0.6); } \\
\text { Hexachlorobenzene (1); Hexachlorocyclopentadiene (50); } \\
\text { Pentachlorophenol (1); Styrene (100); Tetrachloroethene (5); } \\
\text { Toluene(1000); Trichloroethene(5); Xylenes (10,000) }\end{array}$ & $\begin{array}{l}\text { (USEPA, } \\
\text { 2009) }\end{array}$ \\
\hline & & Australia & $\begin{array}{l}\text { Australian Drinking Water } \\
\text { Guidelines }\end{array}$ & Drinking water & $\begin{array}{l}\text { Benzene (1); Carbon tetrachloride (4); 1,2-Dichlorobenzene } \\
\text { (1); 1,3-Dichlorobenzene (20); 1,4-Dichlorobenzene (0.3); } \\
\text { 1,2-Dichloroethane (3); 1.2-Dichloroethene (60); } \\
\text { Dichloromethane (4); Di(2-ethylhexyl)phthalate; etc. }\end{array}$ & $\begin{array}{l}\text { (NRMMC, } \\
\text { 2015) }\end{array}$ \\
\hline & & Australia & $\begin{array}{l}\text { Australian Guidelines for } \\
\text { Water Recycling }\end{array}$ & $\begin{array}{l}\text { Secondary treated } \\
\text { effluent for } \\
\text { augmentationof } \\
\text { drinking water supplies }\end{array}$ & $\begin{array}{l}\text { 1,1-Dichloroethene (30); Coprastanol (0.7); } \\
\text { 1,7-Dimethylxanthine(0.7); Coumarin (0.5); } \\
\text { 2,5-Dihydroxybenzoicacid(7); Diatrizoate Sodium(0.35); } \\
\text { 2,6-di-tert-butyl-1,4-benzoquinone (0.014); Diatrizoic acid } \\
\text { (0.350); 2,6-di-tert-butylphenol (2); Dibutyltin (2); } \\
\text { 4-Chlorophenol (10); Di-n-butyl phthalate (35); } \\
\text { 4-cumylphenol (0.35); Methylene chloride (4); 4-tert } \\
\text { octylphenol (50); Monobutyltin (0.7); } \\
\text { 5-methyl-1Hbenzotriazole (0.007); Naphthalene (70); } \\
\text { Anthracene (150); N-nitrosomorpholine (0.001); } \\
\text { Acetophenone (400); Phenanthrene (150); Benzo(a)pyrene } \\
\text { (0.01); Phenol (150); Benzyl chloride (0.2); Bisphenol A } \\
\text { (200); Pyrene(150); Bromochloromethane (40); Tributyl } \\
\text { phosphate (0.5); Butylated hydroxyanisole; Tributyltin (1); } \\
\text { Caffeine (0.35); Tri(butyl cellosolve) phosphate (50); } \\
\text { Chlorophene(0.35); Cholesterol(7); Triphenylphosphate } \\
\text { (1); 2,4,6-Trinitro-1,3- dimethyl-5-tertbutylbenzene (350); } \\
\text { 4-Acetyl-6-t-butyl-1,1-dimethylindan(7); } \\
\text { 6-Acetyl-1,1,2,4,4,7- hexamethyltetraline (4); Galaxolide } \\
\text { (0.0018); Musk ketone (350); Musk tibetene (0.35); } \\
\text { Pentamethyl-4,6- dinitroindane (0.35) }\end{array}$ & $\begin{array}{l}\text { NRMMC } \\
(2008)\end{array}$ \\
\hline 2. & \multicolumn{6}{|c|}{ Pharmaceuticals and steroid hormones } \\
\hline a. & Antibiotics & Australia & $\begin{array}{l}\text { Australian Guidelines for } \\
\text { Water Recycling }\end{array}$ & $\begin{array}{l}\text { Secondary treated } \\
\text { effluent for } \\
\text { augmentation of } \\
\text { drinking water supplies }\end{array}$ & $\begin{array}{l}\text { Amoxycillin (1.5); Monensin (35); Anhydroerythromycin A } \\
\text { (17.5); Naladixic acid (1000); Azithromycin (3.9); Norflaxin } \\
\text { (400); Cefaclor (250); Penicillin G (1.5); Cephalaxin (35); } \\
\text { Penicillin V (1.5); Chloroamphenicol (175); Roxithromycin } \\
\text { (150); Chlorotetracycline(105); Sulfamethoxazole (35); } \\
\text { Ciproflaxin (250); Sulfamethoxine (35); Clarithromycin } \\
\text { (250); Sulfamethazine (35); Clindamycin (300); } \\
\text { Sulfamethizole (35); Demeclocycline (300); Terramycin } \\
\text { (105); Doxycycline(10.5); Tetracycline(105); Enrofloxacin } \\
\text { (22); Trimethoprim (70); Erythromycin (17.5); Tylosin } \\
\text { (1050) }\end{array}$ & $\begin{array}{l}\text { NRMMC } \\
(2008)\end{array}$ \\
\hline b. & $\begin{array}{l}\text { Non-steroidal } \\
\text { anti-inflammatories }\end{array}$ & Australia & & & $\begin{array}{l}\text { Aspirin (29); Indomethacin (25); Diclofenac (1.8); } \\
\text { Ketoprofen (3.5); Dipyrone (vet) (525); Naproxen (220); } \\
\text { Fenoprofen (450); Tolfenamic acid (vet) (17.5); Ibuprofen } \\
\text { (400) }\end{array}$ & $\begin{array}{l}\text { NRMMC } \\
(2008)\end{array}$ \\
\hline c. & $\begin{array}{l}\beta \text {-adrenergic } \\
\text { blockers }\end{array}$ & Australia & & & $\begin{array}{l}\text { Betaxolol (10); Nadolol (20); Bisoprolol (0.63); Propranolol } \\
\text { (40); Carazolol (0.35); Timolol (10); Metoprolol (25) }\end{array}$ & $\begin{array}{l}\text { NRMMC } \\
(2008)\end{array}$ \\
\hline \multirow[t]{2}{*}{ d. } & $\begin{array}{l}\text { Estrogenic } \\
\text { hormones }\end{array}$ & Australia & & & $\begin{array}{l}\text { 17 } \alpha \text {-estradiol (0.175); Estriol (0.050); } 17 \alpha \text {-ethinyl estradiol } \\
\text { (0.0015); Estrone (0.03); } 17 \beta \text {-estradiol }(0.175) \text {; Mestranol } \\
(0.0025) \text {; Equilenin }(0.030) \text {; Norethindrone }(0.25) \text {; Equilin } \\
(0.030) \text { Progesterone (105) }\end{array}$ & $\begin{array}{l}\text { NRMMC } \\
(2008))\end{array}$ \\
\hline & & $\begin{array}{l}\text { National } \\
\text { Water } \\
\text { Research } \\
\text { Institute } \\
\text { (USA) }\end{array}$ & $\begin{array}{l}\text { Report on public health criteria } \\
\text { for direct potable reuse } \\
\text { systems (2013) }\end{array}$ & $\begin{array}{l}\text { Secondary treated } \\
\text { effluent for direct } \\
\text { potable reuse }\end{array}$ & Estrone $(0.32)$ & $\begin{array}{l}\text { Audenaert } \\
\text { et al. } \\
(2014)\end{array}$ \\
\hline e. & Androgens & Australia & $\begin{array}{l}\text { Australian Guidelines for } \\
\text { Water Recycling }\end{array}$ & $\begin{array}{l}\text { Secondary treated } \\
\text { effluent for }\end{array}$ & Androsterone (14); Testosterone (7) & $\begin{array}{l}\text { NRMMC } \\
(2008)\end{array}$ \\
\hline f. & $\begin{array}{l}\text { General } \\
\text { pharmaceuticals }\end{array}$ & Australia & & $\begin{array}{l}\text { augmentation of } \\
\text { drinking water supplies }\end{array}$ & $\begin{array}{l}\text { Alprazolam (0.25); Fluoxetine (10); Antipyrine (1000); } \\
\text { Gemfibrozil (600); Atorvastatin (5); Iohexol (720); }\end{array}$ & $\begin{array}{l}\text { NRMMC } \\
(2008)\end{array}$ \\
\hline
\end{tabular}


Table 2 (continued)

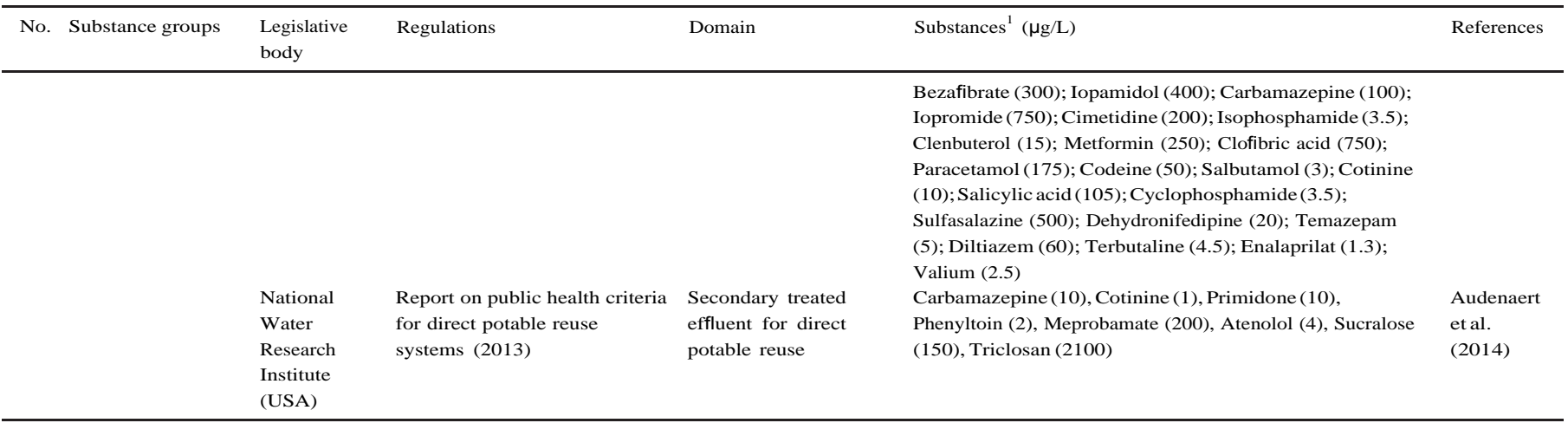

${ }^{1}$ Regulated value (unit: $\mu \mathrm{g} / \mathrm{L}$ ) for each compound is presented in bracket (), for example: Simazine (20) means the required concentration of Simazine is $20 \mu \mathrm{g} / \mathrm{L}$.

content may interfere the adsorption of targeted contaminants. Activated carbon shows a higher preference towards hydrophobic (log $\mathrm{K}_{\mathrm{ow}} \mathrm{N} 4$ ), low molecular weight, slightly positively charged compounds (at $\mathrm{pH}=7-8$ ) and compounds contain aromaticity and $\mathrm{N}$-heterocycles (Table 4).

Activated carbon technologies are preferred by EU countries as an upgrading option for their WWTPs because they can remove most of MPs in municipal WWTP effluents by $80 \%$. In Germany alone, there are 14 WWTPs already adopting PAC/GAC technologies, six WWTPs under construction and three WWTPs under design (Mulder et al., 2015).

\subsubsection{Advanced oxidation processes (AOPs)}

Oxidation methods aim to destruct and transform refractory substances to simpler substances. Since traditional oxidation methods (i.e., $\mathrm{Cl}_{2}, \mathrm{HClO}, \mathrm{H}_{2} \mathrm{O}_{2}, \mathrm{KMnO}_{4}$ and $\mathrm{ClO}_{2}$ ) are ineffective in tackling MPs, only advanced oxidation methods (AOPs) such as $\mathrm{O}_{3}, \mathrm{UV} / \mathrm{H}_{2} \mathrm{O}_{2}, \mathrm{O}_{3} /$ $\mathrm{H}_{2} \mathrm{O}_{2}$, and $\mathrm{UV} / \mathrm{O}_{3}$ are considered in full-scale applications. AOPs presented higher MP removal performance than traditional oxidation methods due to stronger oxidant doses, higher magnitude of free radical compounds hydrogen peroxy $\left(\mathrm{HO}_{2}\right)$ and hydroxyl $(\mathrm{OH})$, and longer contact time (Lee et al., 2009). An oxidation process is often governed by $\mathrm{pH}$, temperature and water chemistry, as well as molecular structure and reactivity of substances $\left(\mathrm{K}_{\mathrm{O} 3}\right.$ and $\left.\mathrm{K}_{\cdot \mathrm{OH}}\right)$. It is advisable that AOPs should be located between two biological processes to reduce the competition with other organic matters in the pre-treatment stage and degrade oxidation by-products in the post-treatment stage.

Beside PAC/GAC, ozonation is a preferable MP removal option in European. It is proven by the number of WWTPs applied ozone technology in Germany and Switzerland, including Wuëri WWTP with the capacity of $350 \mathrm{~m}^{3} / \mathrm{h}$ (Switzerland), Neugut WWTP $875 \mathrm{~m}^{3} / \mathrm{h}$ (Switzerland), Bad Sassendorf WWTP $300 \mathrm{~m}^{3} / \mathrm{h}$ (Germany), Duisburg-Vierlinden WWTP $400 \mathrm{~m}^{3} / \mathrm{h}$ (Germany), Detmold WWTP $300 \mathrm{~m}^{3} / \mathrm{h}$ (Germany) and Schwerte WWTP $1100 \mathrm{~m}^{3} / \mathrm{h}$ (Germany) (Mulder et al., 2015; Audenaert et al., 2014).

While UV alone is not effective in MP elimination, its combination with $\mathrm{H}_{2} \mathrm{O}_{2}$ or $\mathrm{O}_{3}$ can boost the formation of free oxidant radicals and thus, significantly improve the removal rate (Kruithof et al., 2007; Yang et al., 2014). $\mathrm{O}_{3} / \mathrm{UV}$ has the highest oxidation potential and exhibits two-tier barriers for contaminants. This is because $\mathrm{O}_{3}$ has greater UV adsorption capacity by a magnitude of 200 times (at $254 \mathrm{~nm}$ ) than an equivalent amount of $\mathrm{H}_{2} \mathrm{O}_{2}$ does (Sarathy and Mohseni, 2008). However, its high energy consumption inhibits a widespread application of $\mathrm{O}_{3} / \mathrm{UV}$ in reality. To date, $\mathrm{UV} / \mathrm{H}_{2} \mathrm{O}_{2}$ is still the most popular $\mathrm{UV}$-based AOP in large-scale. $\mathrm{H}_{2} \mathrm{O}_{2} / \mathrm{O}_{3}$ is considered the most energy-efficient AOP and is more suitable for water with poor UV light transmission (Lee et al., 2009).

\subsubsection{Membrane processes}

Applicable membrane processes are microfiltration (MF), ultrafiltration (UF), nanofiltration (NF) and reserve osmosis (RO). MF and UF are frequently used to support other MP treatment technologies rather than being used per se because their membrane pores are larger than MP molecular sizes. Filtration and adsorption on the membrane are the key removal mechanisms for membrane processes. The rejection rate depends on both MPs (molecular size and weight, charge and hydrophobicity) and membrane characteristics. With its smallest membrane pore sizes, $\mathrm{RO}$ is the most effective membrane process to remove MPs, even those substances unable to eliminate in MBRs such as diclofenac, carbamazepine, fenoprop, metronidazole and trimethoprime (Hai et al., 2011). Conversely, membrane processes are less sensitive to neutral compounds such as Bisphenol A, NP, NDMA, 17 $\beta$-estradiol, and caffeine (Lee et al., 2009). Consequently, the combination of GAC/PAC and membrane processes are recommended to get a better performance.

\subsubsection{Membrane bioreactors (MBRs)}

MP removal of MBRs largely relies on (i) microorganism consortia to metabolise MPs and (ii) sorption capacity of sludge and (iii) rejection rate of membrane. Extended sludge retention time (SRT) may foster a more diverse microorganism structure which enhances MP removal (Hai et al., 2014). The outperformance of MBR over conventional activated sludge treatment in MP removal is explained in Fig. 1.

The comparison between conventional aerobic processes and MBRs has been carried out by various authors. For example, Bernhard et al. (2006) found that MBRs had a better removal efficiency towards diclofenac, mecoprop and sulfophenylcarboxylates. In a different study, de Wever et al. (2007) confirmed that MBRs significantly improved the elimination of, 6- and 2,7-naphthalene disulfonate (NDSA) and benzothiazole-2-sulfonate. Although MBRs have higher MP removal rates than conventional aerobic biological treatment methods, they are not effective in removing (i) compounds with long, highly branched side chains, (ii) saturated/polycyclic compounds, and (iii) compounds having electron receptive functional groups or sulphate and halogen such as diclofenac, carbamazepine, DEET and trimethoprim (Lee et al., 2009). In addition, microbial community is also subject to toxicity of some recalcitrant substances (Cazes et al., 2014). Despite a huge amount of papers tried different methods to improve the efficiency of MBRs in removing MPs, the majority of them are done at laboratory scale.

\subsection{Environmental considerations}

\subsubsection{Activated carbon adsorption}

The basic principle of adsorption process is to move MPs from liquid phase to solid phase. Compared to advanced oxidation, activated carbon adsorption offers outstanding advantages such (continued on next page) 
Table 3

Case studies for assessment.

\begin{tabular}{|c|c|c|c|c|c|c|c|c|}
\hline No. & Method & Medium & $\begin{array}{l}\text { Treatment } \\
\text { purpose }\end{array}$ & Targeted pollutants & Scale & Treatment train ${ }^{\mathrm{a}}$ & Capacity & Reference \\
\hline 1. & $\mathrm{UV} / \mathrm{H}_{2} \mathrm{O}_{2}$ & $\begin{array}{l}\text { Surface } \\
\text { water }\end{array}$ & $\begin{array}{l}\text { Water } \\
\text { treatment and } \\
\text { supply (organic } \\
\text { removal and } \\
\text { disinfection) }\end{array}$ & $\begin{array}{l}\text { Algae toxins (microcystine), } \\
\text { endocrine disruptors (bisphenol } \\
\text { A), fuel oxygenates (MTBE), } \\
\text { pesticides (atrazine, pyrazon, } \\
\text { diuron, bentazone, bromacil, } \\
\text { dicamba, 2,4-D, TCA, trichlorpyr, } \\
\text { methabenzthiaxuon, } \\
\text { desethyl-desisopropylatrazine), } \\
\text { oxidation by-products (NDMA), } \\
\text { solvents (dioxane), } \\
\text { pharmaceuticals (diclofenac, } \\
\text { ibuprofen) }\end{array}$ & Pilot/Full-scale & $\begin{array}{l}\text { IJssel Lake } \rightarrow \text { Coagulation } \rightarrow \\
\text { Rapid sand filtration } \rightarrow \\
\mathrm{UV} / \mathrm{H}_{2} \mathrm{O}_{2} \text { treatment } \rightarrow \text { GAC } \\
\text { filtration } \rightarrow \text { GAC filtration } \rightarrow \\
\text { Chlorine dioxide dosage } \rightarrow \\
\text { drinking water }\end{array}$ & $\begin{array}{l}\text { Andijk } \\
\text { surface water } \\
\text { treatment } \\
\text { plant } \\
\text { (Holland): } \\
6000 \mathrm{~m}^{3} / \mathrm{h}\end{array}$ & $\begin{array}{l}\text { Kruithof et al. } \\
\text { (2007) }\end{array}$ \\
\hline 2. & $\begin{array}{l}\text { Granular activated } \\
\text { carbon (GAC) }\end{array}$ & $\begin{array}{l}\text { Surface } \\
\text { water }\end{array}$ & Drinking water & Organic MPs (24) & Pilot/Full-scale & N/A & N/A & $\begin{array}{l}\text { Kennedy et al. } \\
\text { (2015) }\end{array}$ \\
\hline 3. & $\mathrm{O}_{3}$ & $\begin{array}{l}\text { Municipal } \\
\text { wastewater }\end{array}$ & $\begin{array}{l}\text { Wastewater } \\
\text { discharge }\end{array}$ & $\begin{array}{l}\text { Biocides and pesticides (10), } \\
\text { pharmaceuticals (24), } \\
\text { transformation products (7), } \\
\text { X-ray contrasting media (6), } \\
\text { corrosion inhibitors (2), } \\
\text { nitrosamines (8) }\end{array}$ & Full-scale & $\begin{array}{l}\text { Primary sedimentation } \rightarrow \\
\text { Activated sludge treatment } \\
\rightarrow \text { Sedimentation } \rightarrow \\
\text { Ozonation } \rightarrow \text { Sand filtration } \\
\rightarrow \text { Discharge }\end{array}$ & $\begin{array}{l}\text { Wüeri } \\
\text { WWTP } \\
\text { (Regensdorf, } \\
\text { Switzerland): } \\
5500 \mathrm{~m}^{3} / \text { day }\end{array}$ & $\begin{array}{l}\text { Hollender et al. } \\
\text { (2009) }\end{array}$ \\
\hline 4. & $\mathrm{O}_{3}$ & $\begin{array}{l}\text { Secondary } \\
\text { treated } \\
\text { wastewater }\end{array}$ & $\begin{array}{l}\text { Reuse for } \\
\text { irrigation, dual } \\
\text { recirculation } \\
\text { and fire fighting }\end{array}$ & $\begin{array}{l}387 \text { substances: nitrosamines } \\
\text { (5), endocrine disrupting } \\
\text { compounds (20), } \\
\text { pharmaceuticals (58), phenoxy } \\
\text { herbicides (11), haloacetic acids } \\
\text { (9), herbicides (51), } \\
\text { organochlorine pesticides (33), } \\
\text { organophosphorus pesticides } \\
\text { (46), other pesticides (25), } \\
\text { synthetic pyrethroids (12), other } \\
\text { compounds (20), phenolics (18), } \\
\text { polycyclic aromatic } \\
\text { hydrocarbons (pahs) (30), } \\
\text { trihalomethanes (5), iodinated } \\
\text { halomethanes (5), } \\
\text { perfluorinated compounds (17), } \\
\text { aldehydes (4), disinfection } \\
\text { by-products (11) }\end{array}$ & Full-scale & $\begin{array}{l}\text { Pre-ozone } \rightarrow \text { Biological } \\
\text { media filtration } \rightarrow \\
\text { Post-ozone } \rightarrow \mathrm{UV} \rightarrow \\
\text { Chlorination }\end{array}$ & $\begin{array}{l}\text { Eastern } \\
\text { Treatment } \\
\text { Plant } \\
\text { (Melbourne, } \\
\text { Australia): } \\
\text { 350 ML/day }\end{array}$ & $\begin{array}{l}\text { Blackbeard et al. } \\
\text { (2016) }\end{array}$ \\
\hline 5. & GAC & $\begin{array}{l}\text { Sewage } \\
\text { wastewater }\end{array}$ & Discharge & $\begin{array}{l}\text { Propranolol, sulfamethoxazole, } \\
\text { mebeverine, thioridazine, } \\
\text { carbamazepine, tamoxifen, } \\
\text { mecoprop, indomethacin, } \\
\text { diclofenac, meclofenamic acid, } \\
\text { monensin }\end{array}$ & Full-scale & $\begin{array}{l}\text { Screens } \rightarrow \text { Primary } \\
\text { sedimentation } \rightarrow \text { Activated } \\
\text { sludge } \rightarrow \text { Secondary } \\
\text { sedimentation } \rightarrow \text { GAC } \rightarrow \\
\text { Discharge }\end{array}$ & $\begin{array}{l}\text { Sewage } \\
\text { treatment } \\
\text { plant } \\
\text { (Swindon, } \\
\text { UK): N/A }\end{array}$ & $\begin{array}{l}\text { Grover etal. } \\
\text { (2011) }\end{array}$ \\
\hline 6. & $\begin{array}{l}\text { Membrane } \\
\text { bioreactors }\end{array}$ & $\begin{array}{l}\text { Municipal } \\
\text { wastewater }\end{array}$ & Reuse & $\begin{array}{l}\text { Steroidal hormones, } \\
\text { xenoestrogens, pesticides, } \\
\text { caffeine and pharmaceuticals } \\
\text { and personal care products } \\
\text { (PPCPs) }\end{array}$ & Full-scale & $\begin{array}{l}\text { Screens } \rightarrow \mathrm{MBR} \rightarrow \mathrm{UV} \rightarrow \\
\text { Storage and reuse }\end{array}$ & $\begin{array}{l}\text { Wolumba, } \\
\text { Bega Valley, } \\
\text { New South } \\
\text { Wales } \\
\text { (Australia) }\end{array}$ & $\begin{array}{l}\text { Trinh et al. } \\
\text { (2012) }\end{array}$ \\
\hline 7. & $\begin{array}{l}\text { Membrane } \\
\text { bioreactors }\end{array}$ & $\begin{array}{l}\text { Hospital } \\
\text { wastewater }\end{array}$ & $\begin{array}{l}\text { Discharge to } \\
\text { WWTP }\end{array}$ & Pharmaceuticals & Full-scale & $\begin{array}{l}\text { Screens } \rightarrow \text { Primary } \\
\text { settlement } \rightarrow \text { MBR } \rightarrow \\
\text { Advanced treatment } \rightarrow \\
\text { Discharge to sewer }\end{array}$ & $\begin{array}{l}\text { Waldbrol } \\
\text { hospital } \\
\text { (Germany): } \\
Q=130 \\
\mathrm{~m}^{3} / \mathrm{d}\end{array}$ & $\begin{array}{l}\text { Beieretal. } \\
\text { (2011) }\end{array}$ \\
\hline 8. & $\begin{array}{l}\text { Membrane } \\
\text { bioreactors }\end{array}$ & $\begin{array}{l}\text { Municipal } \\
\text { wastewater }\end{array}$ & $\begin{array}{l}\text { Wastewater } \\
\text { discharge }\end{array}$ & $\begin{array}{l}\text { Pharmaceuticals (17) and trace } \\
\text { organic pollutants (22) }\end{array}$ & Pilot & $\begin{array}{l}3 \text { different processes: } \\
\text { 1. Integrated fixed-film } \\
\text { activated sludge MBR } \\
\text { (IFAS-MBR)+RO } \\
\text { 2. Moving bed MBR } \\
\text { (MBMBR) + RO } \\
\text { 3. MBR+RO }\end{array}$ & $2.3 \mathrm{~m}^{3} / \mathrm{h}$ & $\begin{array}{l}\text { de la Torre et al. } \\
\text { (2015) }\end{array}$ \\
\hline 9. & $\begin{array}{l}\text { Enzymatic } \\
\text { membrane } \\
\text { bioreactors }\end{array}$ & Wastewater & $\begin{array}{l}\text { Wastewater } \\
\text { discharge }\end{array}$ & Tetracycline & Pilot & N/A & $1043 \mathrm{~L} / \mathrm{m}^{2} . \mathrm{h}$ & $\begin{array}{l}\text { Abejon et al. } \\
\text { (2015) }\end{array}$ \\
\hline 10. & Activated carbon & Wastewater & $\begin{array}{l}\text { Wastewater } \\
\text { discharge }\end{array}$ & $\begin{array}{l}5 \text { organic MPs (diclofenac, } \\
\text { carbamazepine, benzotriazole, } \\
\text { methylbenzotriazole, } \\
\text { formylaminoantipyrine) }\end{array}$ & Pilot & $\begin{array}{l}1 . \\
\text { Coagulation/sedimentation } \\
+ \text { PAC } \\
2 . \\
\text { Coagulation/sedimentation } \\
+ \text { GAC (dual filter) }\end{array}$ & $0.6 \mathrm{~m}^{3} / \mathrm{h}$ & $\begin{array}{l}\text { Meinel et al. } \\
\text { (2014) }\end{array}$ \\
\hline 11. & $\begin{array}{l}\text { Biofiltration + } \\
\text { ozonation }\end{array}$ & $\begin{array}{l}\text { Municipal } \\
\text { wastewater }\end{array}$ & $\begin{array}{l}\text { Wastewater } \\
\text { reuse }\end{array}$ & Antibiotics, EDCs & Pilot & $\begin{array}{l}\text { Secondary effluent } \rightarrow \\
\text { Denitrification biofilter } \rightarrow\end{array}$ & $0.6 \mathrm{~m}^{3} / \mathrm{h}$ & X. Li et al. (2015) \\
\hline
\end{tabular}


Table 3 (continued)

\begin{tabular}{|c|c|c|c|c|c|c|c|c|}
\hline No. & Method & Medium & $\begin{array}{l}\text { Treatment } \\
\text { purpose }\end{array}$ & Targeted pollutants & Scale & Treatment train $^{\mathrm{a}}$ & Capacity & Reference \\
\hline & & & & & & Ozonation reactor $\rightarrow$ & & \\
\hline 12. & $\begin{array}{l}\text { Microfiltration } \\
\text { (MF)+reverse } \\
\text { osmosis (RO) }\end{array}$ & $\begin{array}{l}\text { Municipal } \\
\text { wastewater }\end{array}$ & Water reuse & $\begin{array}{l}\text { Pharmaceuticals (29) and } \\
\text { pesticides (20) }\end{array}$ & Pilot & $\begin{array}{l}\text { Biological aerated filtration } \\
\text { Secondary effluent } \rightarrow \text { UV } \rightarrow \\
\text { Microfiltration } \rightarrow \text { RO }\end{array}$ & $2 \mathrm{~m}^{3} / \mathrm{h}$ & $\begin{array}{l}\text { Rodriguez-Mozaz } \\
\text { et al. (2015) }\end{array}$ \\
\hline 13. & $\begin{array}{l}\text { Ultrafiltration + } \\
\text { activated sludge } \\
\text { reactor }\end{array}$ & $\begin{array}{l}\text { Hospital } \\
\text { wastewater }\end{array}$ & $\begin{array}{l}\text { Wastewater } \\
\text { discharge }\end{array}$ & Pharmaceuticals (21) & Pilot & $\begin{array}{l}\text { Activated sludge (or } \\
\text { biofilm) } \rightarrow \text { Ultrafiltration }\end{array}$ & $0.9 \mathrm{~m}^{3} / \mathrm{h}$ & $\begin{array}{l}\text { Mousaab et al. } \\
\text { (2015) }\end{array}$ \\
\hline 14. & $\begin{array}{l}\text { Ozone }+ \text { activated } \\
\text { carbon(AC) }\end{array}$ & $\begin{array}{l}\text { Synthetic } \\
\text { wastewater }\end{array}$ & $\begin{array}{l}\text { Removal of } \\
\text { diethylphthalate }\end{array}$ & Diethylphthalate & Pilot & Wastewater $\rightarrow \mathrm{AC} \rightarrow \mathrm{O}_{3}$ & $5-20 \mathrm{~L} / \mathrm{h}$ & $\begin{array}{l}\text { Chedeville et al. } \\
\text { (2014) }\end{array}$ \\
\hline 15. & $\begin{array}{l}\text { Nanofiltration } \\
(\mathrm{NF})+\text { solar } \\
\text { photo-Fenton }\end{array}$ & $\begin{array}{l}\text { WWTP } \\
\text { effluent + } \\
\text { spiked } \\
\text { chemicals }\end{array}$ & $\begin{array}{l}\text { Removal of } \\
\text { pharmaceuticals }\end{array}$ & $\begin{array}{l}\text { Pharmaceuticals (5): } \\
\text { carbamazepine, flumequine, } \\
\text { ibuprofen, ofloxacin and } \\
\text { sulfa-methoxazole }\end{array}$ & Pilot & $\begin{array}{l}\text { 1. WWTP effluent } \rightarrow \\
\text { pre-filtration } \rightarrow \text { NF } \\
2 \text {.WWTP effluent } \rightarrow \\
\text { pre-filtration } \rightarrow \text { solar } \\
\text { photo-Fenton }\end{array}$ & $100 \mathrm{~L} / \mathrm{h}$ & $\begin{array}{l}\text { Miralles-Cuevas } \\
\text { et al. (2014) }\end{array}$ \\
\hline 16. & $\begin{array}{l}\text { Enzymatic } \\
\text { membrane reactor } \\
(\mathrm{MBR})\end{array}$ & $\begin{array}{l}\text { Spiked } \\
\text { wastewater }\end{array}$ & $\begin{array}{l}\text { Removal of } \\
\text { tetracycline }\end{array}$ & Tetracycline & Pilot & $\begin{array}{l}\text { Batch experiment: } \\
\text { Wastewater } \rightarrow \text { MBR }\end{array}$ & N/A & $\begin{array}{l}\text { de Cazes et al. } \\
\text { (2014) }\end{array}$ \\
\hline 17. & Powdered PAC & $\begin{array}{l}\text { Municipal } \\
\text { wastewater }\end{array}$ & $\begin{array}{l}\text { Wastewater } \\
\text { discharge }\end{array}$ & $\begin{array}{l}\text { Pharmaceuticals andhormones } \\
\text { (54)+ other emerging } \\
\text { pollutants (59) }\end{array}$ & Pilot & $\begin{array}{l}\text { [Screening } \rightarrow \text { Grit and oil } \\
\text { removal } \rightarrow \text { lamellar settling } \\
\text { unit with coagulation } \rightarrow\end{array}$ & 6-12 m/h & $\begin{array}{l}\text { Mailler et al. } \\
\text { (2015) }\end{array}$ \\
\hline 18. & $\mathrm{UV} / \mathrm{H}_{2} \mathrm{O}_{2}$ & $\begin{array}{l}\text { Municipal } \\
\text { wastewater } \\
\text { (secondary) }\end{array}$ & $\begin{array}{l}\text { Wastewater } \\
\text { reuse }\end{array}$ & $\begin{array}{l}\text { EDCs, herbicides, pesticides, } \\
\text { volatile pesticides, NDMA }\end{array}$ & Pilot & $\begin{array}{l}\text { 3-stage bio-filters] } \rightarrow \text { PAC } \\
\text { Conventional activated } \\
\text { sludge (CAS) treated } \\
\text { wastewater } \rightarrow \text { pre-filter } \rightarrow\end{array}$ & $600 \mathrm{~m}^{3} /$ day & $\begin{array}{l}\text { James et al. } \\
(2014)\end{array}$ \\
\hline 19. & $\begin{array}{l}\text { PAC/Ultrafiltration } \\
\text { (UF) }\end{array}$ & $\begin{array}{l}\text { Municipal } \\
\text { wastewater }\end{array}$ & $\begin{array}{l}\text { Wastewater } \\
\text { discharge }\end{array}$ & $\begin{array}{l}\text { Benzotriazole; Carbamazepine; } \\
\text { Diclofenac; Mecoprop; } \\
\text { Sulfamethoxazole; }\end{array}$ & Pilot & $\begin{array}{l}\mathrm{MF} \rightarrow \mathrm{RO} \rightarrow \mathrm{H}_{2} \mathrm{O}_{2} / \mathrm{UV} \\
\text { [Screening } \rightarrow \text { Grit removal } \\
\rightarrow \text { Fine screening } \rightarrow \text { SBR } \\
\text { with nutrient removal] } \rightarrow \\
\text { PAC/UF }\end{array}$ & $\begin{array}{l}20-80 \\
\mathrm{~L} / \mathrm{m}^{2} \cdot \mathrm{h}\end{array}$ & $\begin{array}{l}\text { Lowenberg et al. } \\
\text { (2014) }\end{array}$ \\
\hline
\end{tabular}

Note: N/A: non-assessment.

a Processes presented in bracket [...] are pre-treatment for the experiment's influent.

consumption and no by-product formation. However, spent adsorbents which contain adsorbed MPs are considered hazardous waste and must be managed properly.

3.3.1.1. GAC. For GAC filters, MP removal is greatly affected by the frequency of adsorbent replacement or regeneration. Despite GAC can be reused, its regeneration demands high energy demand for production of hot steam to desorb high-molecular-weight pollutants. The desorbed stream should be managed as hazardous waste. In addition, due to the weakly attachment of some MPs on GAC as well as competition from NOMs, several MPs such as MTBE tends to desorb back to the solution (Creek and Davidson, 2000).

3.3.1.2. PAC. Unlike GAC, PAC cannot be regenerated. Therefore, extending PAC life-time by recycling it into biological treatment tanks is crucial to utilise its full capacity. The return of PAC into aerobic tanks may boost the MP removal performance by 5-10\% (Grover et al., 2011). Nevertheless, PAC addition (10-20 g PAC/ $\left.\mathrm{m}^{3}\right)$ increased the sludge volume by 5$10 \%$ (Margot et al., 2013). Exhausted PAC must be separated from wastewater by sand filtration or membrane processes. To reduce desorption of contaminants from adsorbents back to liquid phase, membrane processes are preferred over sand filtration although sand filtration post-treatment is more economical (Margot et al., 2013). Wasted PAC must be finally incinerated or disposed as hazardous waste.

\subsubsection{Advanced oxidation processes (AOPs)}

The ultimate concern in AOP applications is that AOPs do not completely get rid of MPs but transform them into simpler compounds (Verlicchi et al., 2015). Unfortunately, some of those compounds react with other chemicals (bromides) or DOC presented in wastewater and create new intermediates such as bromate, nitrosamines, chloral hydrate and carbonyl (Blackbeard et al., 2016; X. Li et al., 2015). The transformation process is unpredictable as it is strongly influenced by specific water matrix, $\mathrm{pH}$ and DOC content (Hollender et al., 2009).

3.3.2.1. Ozonation and ozone-based AOPs. After an ozonation process, Blackbeard et al. (2016) observed a formation of atrazine by-products (desisopropyl atrazine and desethyl atrazine), a two-fold increase in NDMA (from $15.3 \mathrm{ng} / \mathrm{L}$ to $31.4 \mathrm{ng} / \mathrm{L}$ ), and especially, a 40-time amplification of bisphenol A. Some transformation products may be more toxic than their precursors. X. Li et al. (2015) found the inhibitory rate on Vidua fischeri increased from 9\% (prior to ozonation) to 15\% (after ozonation). Further treatments such as sand/biological filtration (X. Li et al., 2015) or UV (Siegrist and Joss, 2012) are exploited to reduce these compounds as well as their toxicology.

Ozone production process consumed a huge amount of energy with a low efficiency of conversion when the maximum concentration of ozone produced in air or oxygen was only $1-2 \%$ or $4-8 \%$, respectively (Derco et al., 2015). Ozone generation firstly required an energy-intensive production of absolutely dry air or oxygen (0.01-0.015 $\mathrm{kWh}$ per $\mathrm{m}^{3}$ of pure oxygen produced) (Hollender et al., 2009). In addition, the energy demand for $1 \mathrm{~kg}$ of $\mathrm{O}_{3}$ produced from process gas having 100-170 g $\mathrm{O}_{3}$ per $\mathrm{m}^{3}$ is $12 \mathrm{kWh}$, excluding the oxygen production process (Hollender et al., 2009). About 85\% of the consumed energy is wasted as heat and needed to be eradicated to protect the reactor from overheating.

Ozone has a short life cycle. While its theoretical half-life time is 38 days in air or $15-20 \mathrm{~min}$ in water at $20-25^{\circ} \mathrm{C}$; its actual degradation rate can range from seconds to hours, depending on water temperature, $\mathrm{pH}, \mathrm{DOC}$ and ozone scavengers (Lenntech, 2016). As such, $\mathrm{O}_{3}$ must be generated onsite (Derco et al., 2015). Additionally, ozone handling yields potential fire hazards and toxicity issues. Because ozone is a strong oxidant, it may cause respiratory problems for workers when being exposed to the peak concentration of 0.1 pprffintiptsehfinner(safye) 
Table 4

Treatment methods, physical-chemical characteristics of substances, removal mechanisms and efficiency.

\begin{tabular}{|c|c|c|c|c|c|c|c|c|}
\hline \multirow[b]{2}{*}{ Processes } & \multirow{2}{*}{$\begin{array}{l}\text { Removal } \\
\text { mechanisms }\end{array}$} & \multicolumn{2}{|c|}{ Operational factors } & \multicolumn{4}{|c|}{ Physical-chemical characteristics of substances } & \multirow[b]{2}{*}{ References } \\
\hline & & Factors & Influence & Characteristics & $\begin{array}{l}\text { High efficiency } \\
(\geq 70 \%)\end{array}$ & $\begin{array}{l}\text { Medium efficiency } \\
(40-70 \%)\end{array}$ & $\begin{array}{l}\text { Low efficiency } \\
(\leq 40 \%)\end{array}$ & \\
\hline \multirow[t]{5}{*}{$\begin{array}{l}\text { Activated carbon } \\
\text { adsorption }\end{array}$} & $\begin{array}{l}\text { - Filtration } \\
\text { - Adsorption }\end{array}$ & - Dosage & $\begin{array}{l}\text { - Leading operational fac- } \\
\text { tor } \\
\cdot \text { Optimal PAC dose } \\
=10-20 \mathrm{mg} / \mathrm{L} \text {. Higher } \\
\text { dosages }(40-50 \mathrm{mg} / \mathrm{L}) \\
\text { may result in higher re- } \\
\text { moval rate. }\end{array}$ & $\begin{array}{l}\text { - Charge at } \mathrm{pH}= \\
\text { 7-8: most im- } \\
\text { portant factor }\end{array}$ & $\begin{array}{l}\text { Slightly positively charged } \\
\text { compounds: atenolol, propranolol, } \\
\text { trimethoprim, ciprofloxacin, } \\
\text { norfloxaci }\end{array}$ & & $\begin{array}{l}\text { Slightly negatively charge } \\
\text { compounds: naproxen, diclofenac, } \\
\text { sulfamethoxazole }\end{array}$ & $\begin{array}{l}\text { Mailler et al. } \\
\text { (2015), Altmann } \\
\text { et al. (2014) }\end{array}$ \\
\hline & & - Contact time & $\begin{array}{l}\text { Higher contact time, } \\
\text { higher efficiency } \\
\text { · Optimal } \geq 30 \text { min }\end{array}$ & $\begin{array}{l}\text { Hydrophobicity } \\
\text { (Kow): an im- } \\
\text { portant factor } \\
\text { for neutral } \\
\text { compounds }\end{array}$ & $\begin{array}{l}\text { LogKow N 4: tamoxifen, } \\
\text { indomethacine, diclofenac, } \\
\text { meclofenamic acid }\end{array}$ & 2.5 b LogKow b 4: monensin & $\begin{array}{l}\text { LogKow b 2.5: sulfamethoxazole, } \\
\text { propranolol }\end{array}$ & $\begin{array}{l}\text { Mailler et al. } \\
\text { (2015), Altmann } \\
\text { et al. (2014) }\end{array}$ \\
\hline & & $\cdot \mathrm{pH}$ & $\begin{array}{l}\text { - Affect the charge of hy- } \\
\text { drophilic compounds } \\
\text { - OptimalpH }=7-9\end{array}$ & $\begin{array}{l}\text { Acid dissocia- } \\
\text { tion constants } \\
\left(\mathrm{pK}_{\mathrm{a}}\right)\end{array}$ & & & $\mathrm{pH} \mathrm{N} \mathrm{pKa:} \mathrm{sulfamethoxazole}$ & Nam et al. (2014) \\
\hline & & $\begin{array}{r}\text { Wastewater } \\
\text { composition }\end{array}$ & $\begin{array}{l}\text { Other organic matters } \\
\text { may compete adsorption } \\
\text { sites with MPs (DOC } \\
\leq 5-10 \mathrm{mg} / \mathrm{L} \text { ) }\end{array}$ & $\begin{array}{l}\text { - Molecular size: } \\
\text { large } \\
\text { molecular-size } \\
\text { compounds } \\
\text { show less com- } \\
\text { petition with } \\
\text { natural matters }\end{array}$ & Lower molecular size & & $\begin{array}{l}\text { Large molecular size: erythromycin, } \\
\text { roxithromycin }\end{array}$ & $\begin{array}{l}\text { Mailler et al. } \\
\text { (2015) }\end{array}$ \\
\hline & & & & $\begin{array}{l}\text { Structure and } \\
\text { functional } \\
\text { group: polariz- } \\
\text { ability or struc- } \\
\text { ture of the } \\
\text { compound }\end{array}$ & $\begin{array}{l}\text { Compounds contain aromaticity } \\
\text { and N-heterocycles: ofloxacin, } \\
\text { diclofenac }\end{array}$ & Saccharin, DEHP, bisphenol A & $\begin{array}{l}\text { Halogenated aliphatic: iodinated } \\
\text { contrast media, acesulfame, sucralose, } \\
\text { triclosan, perfluorooctane sulfonate, } \\
\text { nonylphenol }\end{array}$ & $\begin{array}{l}\text { Mailleretal. } \\
\text { (2015) }\end{array}$ \\
\hline \multirow[t]{3}{*}{$\begin{array}{l}\text { Oxidation \& } \\
\text { advanced } \\
\text { oxidation } \\
\text { processes }\left(\mathrm{O}_{3}\right. \\
\text { and } \mathrm{O}_{3} \text {-based } \\
\text { processes, } \\
\left.\mathrm{UV} / \mathrm{H}_{2} \mathrm{O}_{2}\right)\end{array}$} & · Oxidation & - Dosage & $\begin{array}{l}\text { - Higher dosage, higher re- } \\
\text { moval rate } \\
\text { - Can form by-products } \\
\text { - } \mathrm{O}_{3} \text { is less reactive to- } \\
\text { wards organic and inor- } \\
\text { ganic matters } \\
\text { - Dosage }=0.2-0.6 \mathrm{mg} \\
\mathrm{O}_{3} / \mathrm{mg} \text { DOC or } 3-8 \mathrm{mg} \\
\mathrm{O}_{3} / \mathrm{L}\end{array}$ & $\begin{array}{l}\text { - Compound } \\
\text { structure }\end{array}$ & $\begin{array}{l}\text { Contains unsaturated double bonds } \\
\text { (trimethoprim, carbamazepine) } \\
\text { and/or electron donating properties } \\
\text { (diclofenac, macrolides and } \\
\text { sulfonamide antibiotics) }\end{array}$ & $\begin{array}{l}\text { Mostly alkyl aromatics, } \\
\text { secondary amines, thioester } \\
\text { and anisol }\end{array}$ & $\begin{array}{l}\text { Mostly primary amines and nitro } \\
\text { groups, amides, saturated aliphatic or } \\
\text { halogenated aliphatic: bezafibrate }\end{array}$ & $\begin{array}{l}\text { Blackbeard et al. } \\
\text { (2016), Luo et al. } \\
\text { (2014), Altmann } \\
\text { et al. (2014), } \\
\text { Margot et al. } \\
\text { (2013) }\end{array}$ \\
\hline & & $\cdot \mathrm{pH}$ & $\begin{array}{l}\text { - Influence the reactivity } \\
\text { of } \mathrm{O}_{3} \text { and } \bullet \mathrm{OH}\end{array}$ & $\begin{array}{l}\cdot \text { Reactivity with } \\
\mathrm{O}_{3}\left(\mathrm{k}_{\mathrm{O}}\right) \text { or } \bullet \mathrm{OH} \\
\text { radical }(\mathrm{k} \cdot \mathrm{OH})\end{array}$ & $\begin{array}{l}\text { Chemicals with high reaction rates } \\
\text { with ozone }\end{array}$ & $\begin{array}{l}\text { Chemicals with moderate } \\
\text { reaction rates with ozone } \\
\text { and/or } \bullet \text { OH radical }\end{array}$ & $\begin{array}{l}\text { Chemicals with lower reaction rates } \\
\text { with ozone and/or } \bullet \text { OH radical }\end{array}$ & $\begin{array}{l}\text { Blackbeard et al. } \\
\text { (2016) }\end{array}$ \\
\hline & & $\begin{array}{l}\text { Interfering } \\
\text { ions and } \\
\text { wastewater } \\
\text { composition }\end{array}$ & $\begin{array}{l}\text { - Compete with targeted } \\
\text { MPs }\end{array}$ & & $\begin{array}{l}\mathrm{k}_{\mathrm{O} 3} \mathrm{~N} 10^{5} \mathrm{M}^{-1} \mathrm{~s}^{-1} \text { : triclosan, } \\
\text { propranolol, trimethoprim, } \\
\text { carbamazepine, sulfamethoxazole } \\
10^{5} \mathrm{M}^{-1} \mathrm{~s}^{-1} \mathrm{~N}_{\mathrm{o} 3} \geq 10 \mathrm{M}^{-1} \mathrm{~s}^{-1} \text { : } \\
\text { tramadol, venlafaxine, metoprolol, } \\
\text { atenolol }\end{array}$ & $\begin{array}{l}\text { kоз } \mathrm{b} 10 \mathrm{M}^{-1} \mathrm{~s}^{-1} \text { and } \mathrm{k} \cdot \mathrm{oH} \\
\geq 5 \times 10^{9} \mathrm{M}^{-1} \mathrm{~s}^{-1} \text { : } \\
\text { oxpazam, DEET, phenytoin } \\
\text { and primidone }\end{array}$ & $\begin{array}{l}10^{9} \mathrm{M}^{-1} \mathrm{~s}^{-1} \mathrm{~b} \text { k.oн b } 5 \times 10^{9} \mathrm{M}^{-1} \\
\mathrm{~s}^{-1}: 2,4-\mathrm{D} \text {, atrazine, simazine, } \\
\text { iopromide, } \\
\text { k.oH } \mathrm{N} 10^{9} \mathrm{M}^{-1} \mathrm{~s}^{-1} \text { : tris } \\
\text { (chloroethyl)phosphate, } \\
\text { perfluorooctanoic acid, tris } \\
\text { (chloropropyl) phosphate isomers, } \\
\text { tris (dichloro-propyl) phosphate, io- } \\
\text { dinated contrast media }\end{array}$ & \\
\hline $\begin{array}{l}\text { Membrane } \\
\text { processes }\end{array}$ & $\begin{array}{l}\text { - Filtration } \\
\text { - Adsorption }\end{array}$ & $\begin{array}{l}\text { - Membranes: } \\
\text { molecular }\end{array}$ & $\begin{array}{l}\text { Polyamide often have a } \\
\text { better rejection rate }\end{array}$ & $\begin{array}{l}\text { - Molecular } \\
\text { weight and size }\end{array}$ & $\begin{array}{l}\text { RO: } \mathrm{MW} \geq 200 \mathrm{~g} / \mathrm{mol} \text { : most } \\
\text { pharmaceutical compounds, }\end{array}$ & & $\begin{array}{l}\text { Low MW (b200 g/mol): } \\
\text { acetaminophen, gemfibrozil, }\end{array}$ & $\begin{array}{l}\text { Siegrist and Joss } \\
\text { (2012), }\end{array}$ \\
\hline
\end{tabular}


Table 4 (continued)

\begin{tabular}{|c|c|c|c|c|c|c|c|c|}
\hline \multirow{2}{*}{ Processes } & \multirow{2}{*}{$\begin{array}{l}\text { Removal } \\
\text { mechanisms }\end{array}$} & \multicolumn{2}{|l|}{ Operational factors } & \multicolumn{4}{|c|}{ Physical-chemical characteristics of substances } & \multirow[b]{2}{*}{ References } \\
\hline & & Factors & Influence & Characteristics & $\begin{array}{l}\text { High efficiency } \\
(\geq 70 \%)\end{array}$ & $\begin{array}{l}\text { Medium efficiency } \\
(40-70 \%)\end{array}$ & $\begin{array}{l}\text { Low efficiency } \\
(\leq 40 \%)\end{array}$ & \\
\hline \multirow[t]{3}{*}{$\begin{array}{l}\text { (nanofiltration } \\
\text { (NF), reverse } \\
\text { osmosis (RO) }\end{array}$} & & $\begin{array}{l}\text { weight cut-- } \\
\text { off, pore size, } \\
\text { surface } \\
\text { charge, } \\
\text { hydrophobici- } \\
\text { ty, surface } \\
\text { roughness }\end{array}$ & $\begin{array}{l}\text { ( } 57-91 \%) \\
\text { Surface charge of a } \\
\text { membrane will affect the } \\
\text { removal of negatively } \\
\text { charged compounds }\end{array}$ & & $\begin{array}{l}\text { macrolides, cholesterol and some } \\
\text { pesticides (tertbutylazine, diazinon, } \\
\text { MCPA and mecoprop) }\end{array}$ & & mefenamic acid & $\begin{array}{l}\text { Rodriguez-Mozaz } \\
\text { et al. (2015) }\end{array}$ \\
\hline & & $\begin{array}{l}\text { Water: } \mathrm{pH} \\
\text { ionic strength, } \\
\text { hardness, or- } \\
\text { ganic matter }\end{array}$ & $\begin{array}{l}\text { Increased } \mathrm{pH} \text { will in- } \\
\text { crease negative surface } \\
\text { charges, leading to } \\
\text { higher rejection rate }\end{array}$ & $\begin{array}{l}\cdot \text { Charge } \\
\cdot \text { Acid disassocia- } \\
\text { tion constant } \\
\left(\mathrm{K}_{\mathrm{a}}\right)\end{array}$ & $\begin{array}{l}\text { Negatively charged compounds } \\
\text { pH N pKa: ibuprofen }\end{array}$ & & $\begin{array}{l}\text { Non-charged compounds will depend } \\
\text { mainly on adsorption }\end{array}$ & $\begin{array}{l}\text { Siegrist and Joss } \\
(2012) \\
\text { Siegrist and Joss } \\
(2012)\end{array}$ \\
\hline & & & & $\begin{array}{l}\text { Hydrophobicity } \\
(\log D)\end{array}$ & $\begin{array}{l}\text { High hydrophobicity }(\log D \mathrm{~N} 3.2) \text { : } \\
\text { estrone, nonylphenol, triclosan }\end{array}$ & & $\begin{array}{l}\text { Log D b 3.2: Substance removal } \\
\text { depends on its biodegradability } \\
\text { anticorrosive benzotriazoles, } \\
\text { carbamazepine }\end{array}$ & $\begin{array}{l}\text { Tadkaew et al. } \\
\text { (2011) }\end{array}$ \\
\hline \multirow[t]{4}{*}{$\begin{array}{l}\text { Membrane } \\
\text { bioreactors }\end{array}$} & $\begin{array}{l}\text { - Volatilisation } \\
\text { - Biodegradation } \\
\text { - Oxidation }\end{array}$ & $\begin{array}{l}\text { Sludgereten- } \\
\text { tion time }\end{array}$ & $\begin{array}{l}\text { Longer SRT may lead to } \\
\text { higher removal rate (SRT } \\
=10 \text {-80 days) }\end{array}$ & $\begin{array}{l}\text { - Volatility } \\
\text { (Henry constant } \\
\left.\mathrm{k}_{\mathrm{H}}\right)\end{array}$ & $\mathrm{k}_{\mathrm{H}}=10^{-2}-10^{-3}:$ celestolide & & $\begin{array}{l}\text { Some pharmaceuticals, estrogens, } \\
\text { fragrance (tonalide, galaxolide) }\end{array}$ & Luo et al. (2014) \\
\hline & $\begin{array}{l}\text { - Filtration } \\
\text { - Adsorption }\end{array}$ & $\begin{array}{l}\text { Other opera- } \\
\text { tional } \\
\text { parameters }\end{array}$ & $\begin{array}{l}\text { It is recommended to } \\
\text { read more in the review } \\
\text { of Hai et al. (2014) for }\end{array}$ & $\begin{array}{l}\text { Hydrophobicity } \\
(\log \text { D) }\end{array}$ & $\begin{array}{l}\text { High hydrophobicity ( } \log \text { D N 3.2): } \\
\text { estrone, nonylphenol, triclosan }\end{array}$ & & $\begin{array}{l}\text { Log D b 3.2: depend on its } \\
\text { biodegradability: anticorrosive } \\
\text { benzotriazoles, carbamazepine }\end{array}$ & $\begin{array}{l}\text { Tadkaewetal. } \\
\text { (2011) }\end{array}$ \\
\hline & & & $\begin{array}{l}\text { more thorough under- } \\
\text { standings since results } \\
\text { from different experi- } \\
\text { ments are very }\end{array}$ & $\begin{array}{l}\text { - Molecular } \\
\text { weight (MW) }\end{array}$ & $\begin{array}{l}\text { MW N } 300 \mathrm{~g} / \mathrm{mol} \text { : most } \\
\text { pharmaceutical compounds, } \\
\text { macrolides, cholesterol and } \\
\text { pesticides }\end{array}$ & & $\begin{array}{l}\text { MW b } 300 \mathrm{~g} / \mathrm{mol} \text { : depends on } \\
\text { biodegradability }\end{array}$ & $\begin{array}{l}\text { Tadkaew etal. } \\
\text { (2011) }\end{array}$ \\
\hline & & & contradictory. & - Biodegradability & $\begin{array}{l}\text { Linear, with short side chains; } \\
\text { unsaturated aliphatic compounds; } \\
\text { have electron donor functional } \\
\text { groups: steroidal hormones } \\
\text { (androstenedione, androsterone, } \\
\text { etiocholanolone, dihydrotestosterone, } \\
\text { testosterone, 17ß-estradiol, estriol), } \\
\text { xenoestrogens (estrone, bisphenol A, } \\
\text { propylparaben), pharmaceuticals } \\
\text { and PCPs (atenolol, atorvastatin, } \\
\text { DEET, ibuprofen, ketoproen, } \\
\text { metformin, naproxen, triclosan, } \\
\text { caffeine, o-hydeoxyAatorvastatin, } \\
\text { paracetamol) }\end{array}$ & $\begin{array}{l}\text { Contain both electron donor } \\
\text { functional groups and } \\
\text { electron receptive functional } \\
\text { groups: omeprazole, } \\
\text { triameteren, trimethoprim, } \\
\text { amtriptyline, gemfibozil, } \\
\text { sulfamethoxazole, } \\
\text { triclocarban }\end{array}$ & $\begin{array}{l}\text { Long with highly branched side } \\
\text { chains; saturated or polycyclic } \\
\text { compounds; have electron receptive } \\
\text { functional groups or sulfate and } \\
\text { halogen: carbamazepine, dilantin, } \\
\text { phen, linuron, atrazine, DEET, } \\
\text { meprobamate, diazepam, diclofenac, } \\
\text { fluoxetin }\end{array}$ & $\begin{array}{l}\text { Trinh et al. } \\
\text { (2012), Tadkaew } \\
\text { et al. (2011) }\end{array}$ \\
\hline
\end{tabular}




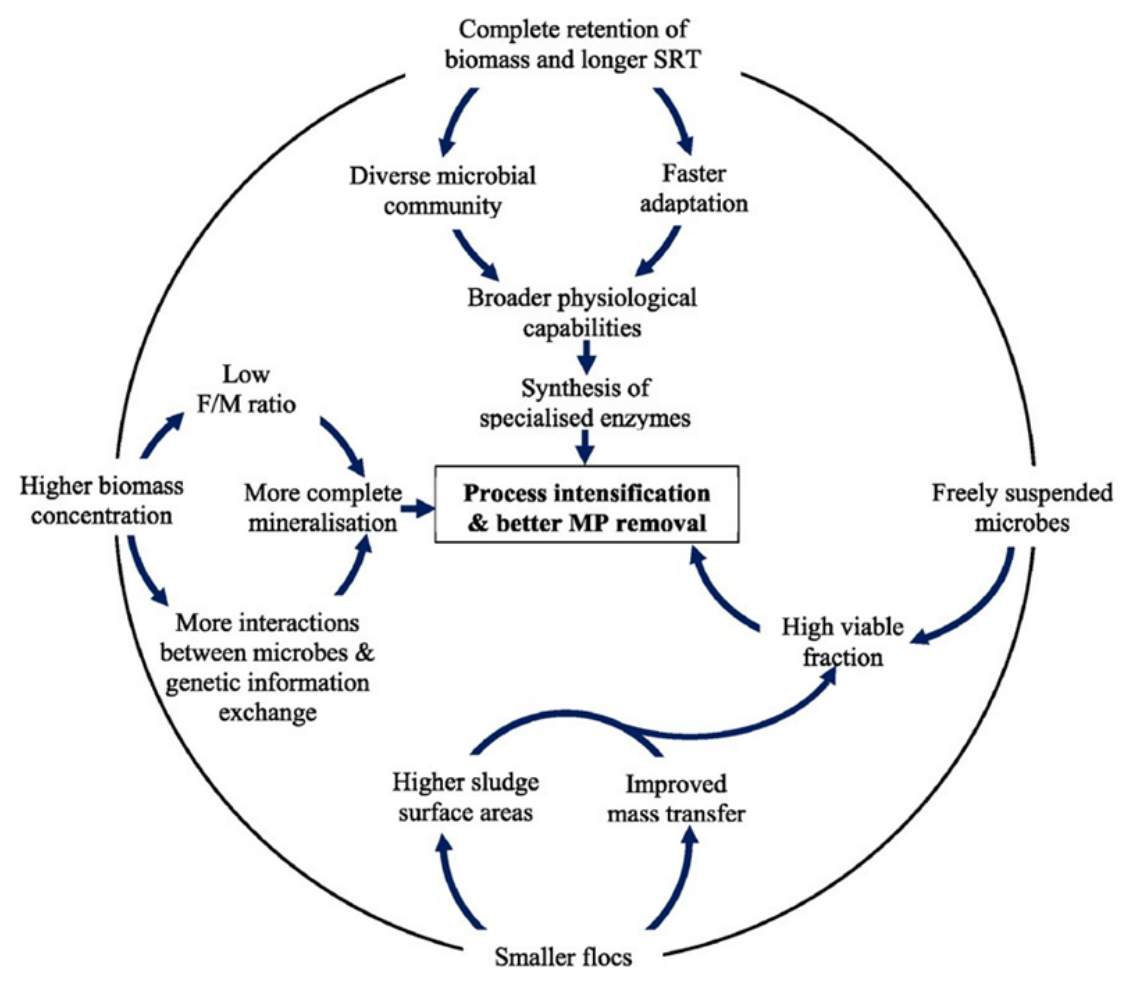

Fig. 1. Improved MP removal mechanisms of MBR in comparison with conventional activated sludge treatment (adapted from (Hai et al., 2014)).

Work Australia, 2015). Thus, sufficient training for people working with ozone is necessary.

Furthermore, despite $\mathrm{O}_{3}$ has a high solubility in water $(109 \mathrm{mg} / \mathrm{L}$ at $25^{\circ} \mathrm{C}$ ), its highly reactivity with pollutants and itself makes it difficult to get a homogenously dissolved ozone concentration. This challenge demands a strong mixing power to maximise contact between oxidants and targeted substances in a short time. As Hollender et al. (2009) calculated, the power rate for treating $1 \mathrm{~m}^{3}$ of wastewater with DOC concentration of $5 \mathrm{~g} / \mathrm{m}^{3}$ and ozone dose of $0.6 \mathrm{~g} \mathrm{O}_{3} / \mathrm{m}^{3}$ is $0.035 \mathrm{kWh}$. This value is slightly lower than those in a Swiss study of $0.06 \mathrm{kWh} / \mathrm{m}^{3}$ for the dose of $5 \mathrm{~g} \mathrm{O}_{3} / \mathrm{m}^{3}$ (Joss et al., 2008).

3.3.2.2. $\mathrm{UV} / \mathrm{H}_{2} \mathrm{O}_{2}$. $\mathrm{H}_{2} \mathrm{O}_{2}$ has several advantages over $\mathrm{O}_{3}$ when it is more stable and can be kept onsite for a long period of time prior to usage (Lee et al., 2009). Kruithof et al. (2007) studied the application of UV/ $\mathrm{H}_{2} \mathrm{O}_{2}$ for both disinfection and MP removal in the Andijk surface water treatment plant and found that the corresponding UV dosage for MP removal was much higher $\left(540 \mathrm{~mJ} / \mathrm{cm}^{2}\right)$ than those required for disinfection $\left(120 \mathrm{~mJ} / \mathrm{cm}^{2}\right)$ with the optimal peroxide dose of $6 \mathrm{mg} / \mathrm{L}$. This treatment process was proven to be robust and reliable. It can also reduce the formation of oxidation by-product NDMA to under $10 \mathrm{ppt}$ (Sarathy and Mohseni, 2008). Nevertheless, the residual $\mathrm{H}_{2} \mathrm{O}_{2}$ must be removed before discharging.

\subsubsection{Membrane processes}

Reserve osmosis has several critical pitfalls. Membrane fouling is the most serious challenge in membrane operation. To control membrane fouling, chemical cleaning by chloramine is necessary to remove fouling layers. The reaction between the disinfectant chloramine and NDMAprecursors in water could result in formation of NDMA (Siegrist and Joss, 2012). Moreover, RO often requires a substantial amount of power to pump water through membrane pores.

Last but not least, RO often produce a large volume of highly concentrated waste stream which enquires further treatment and disposal (Siegrist and Joss, 2012). The waste stream can take account of $35 \%$ of the influent flow and is 4-10 times more concentrated than the influent concentration (Rodriguez-Mozaz et al., 2015; Chelme-Ayala et al.,
2009). Treatment of concentrate still remains a big challenge for operators. Although some advanced oxidation methods were tested for brine treatment, their practical applications are not economically feasible. Therefore, current management method is still to discharge to waste management or evaporating facilities (Rodriguez-Mozaz et al., 2015).

Since the most challenging operation issues of RO are severe membrane fouling and high energy consumption, NF can be a low-pressure alternative with a comparable efficiency (Yangali-Quintanilla et al., 2011).

\subsubsection{Membrane bioreactor (MBR) processes}

The most outstanding advantage of MBRs is the complete elimination of many MPs from the water rather than being transferred to a different phase (as in activated carbon processes), concentrated waste stream (membrane processes) or by-products (AOPs). Nevertheless, MBR may also produce some biotransformation products. MBRs can also provide an effluent free of microorganisms and suspended solids with low concentration of BOD. Conversely, the main concern of using MBR is also membrane fouling. The presence of MPs may contribute to higher degree of irreversible membrane fouling (C. Li et al., 2015). The second issue relating to MBR application is the treatment of wastewater concentrate and sludge that contain MPs. Lastly, nearly 85\% of energy consumed in MBR treatment plants is related to aeration and pumping (Hai et al., 2014).

\subsubsection{Summary}

The environmental consideration of advanced treatment methods are summarised in Table 5 .

\subsection{Technological considerations}

Five technological aspects are considered in this section, including (i) ease of construction and set-up, (ii) flexibility to adapt to the fluctuation in influent water characteristics and flow-rate, (iii) reliability of removal efficiency, (iv) simplicity of operation and (v) maintenance requirements. 


\subsubsection{Activated carbon}

3.4.1.1. GAC. GAC has a long history of application in real-world water treatment plants. It is a simple, flexible and reliable treatment method. GAC can be (1) added into existing filters as an additional layer or (2) worked in a separate GAC filter. Two important parameters of GAC operation are breakthrough capacity and operating time (Lee et al., 2009). With an empty bed contact time of 20 mins, theoretically operating duration of hydrophobic and hydrophilic compounds before saturation are 70,000 and 2000-3000 bed volumes, respectively (Lee et al., 2009). However, in reality, this figure is much lower as materials are deteriorated gradually as number of cycles increased and competing organic matters. The remarkable operational problems of GAC filters are clogging of backwash and surface wash nozzles. Its remediation measures are clearly presented in the literature.

3.4.1.2. PAC. Similar to GAC, PAC is very flexible when it can be added into existing infrastructures (for example, biological tanks) or into a new contacting tank (Lowenberg et al., 2014). Its simplicity in operation is well documented. In spite of this, there are some troubles in the procedure of adding PAC into water. PAC is often stored onsite in silos and then being introduced to a dosage system where PAC is mixed with water before being supplied to treatment tanks. Due to the abrasion nature of the slurry PAC mixture, transporting pipelines can be eroded and clogged (Mulder et al., 2015). In addition, fire hazards from PAC dust should be taken into account.

Last but not least, separation of exhausted PAC is problematic for plant operators. There are two common methods to remove PAC sand filters and UF. Sand filters are often cheaper to operate but UF is more effective to prevent desorption of MPs from PAC back to water (Lowenberg et al., 2014).

\subsubsection{Advanced oxidation}

3.4.2.1. $\mathrm{O}_{3}$ and $\mathrm{O}_{3}$-based AOPs. As analysed in Section 3.3.2, the ozone generation process has a very low productivity; therefore, key technical considerations of ozone treatment are to remain (i) a very effective mass transfer from gas to liquid phase and (ii) an efficient contacting chamber. Ozonation is correspondingly subject to the presence of $\mathrm{O}_{3}$ scavengers, so its removal performance can be deteriorated significantly when influent BOD, COD and SS increased (Environmental Technology Initiative, 2016).

Ozonation processes usually require complicated equipment to produce, control and post-treatment of residual ozone in off-gas. Several operational issues such as (i) feed air quality, (ii) a constant and stable coolant supply to counteract ozone generator over-heating, (iii) regular inspection and maintenance of ozone generators necessitate advanced operational skills (Environmental Technology Initiative, 2016). Therefore, it may not be feasible in small plants with non-permanent staff (Margot et al., 2013).

3.4.2.2. $\mathrm{UV} / \mathrm{H}_{2} \mathrm{O}_{2}$. Compared to $\mathrm{O}_{3}-\mathrm{AOPs}, \mathrm{UV} / \mathrm{H}_{2} \mathrm{O}_{2}$ requires less investment but it involves a specially designed reactor to accommodate UV. This method is not suitable for water with low UV transmission because $\mathrm{H}_{2} \mathrm{O}_{2}$ has a low UV absorbance capacity (Lee et al., 2009). The use of UV/ $\mathrm{H}_{2} \mathrm{O}_{2}$ need to consider the following factors (KFR, 2011):

- UV dose and $\mathrm{H}_{2} \mathrm{O}_{2}$ dose: these are crucial factors for the reactor design. The applied doses depend on UV lamp output, hydraulic conditions and residence time.

- The choice among different technologies medium pressure $\mathrm{UV} / \mathrm{H}_{2} \mathrm{O}_{2}$, low pressure $\mathrm{UV} / \mathrm{H}_{2} \mathrm{O}_{2}$ and dielectric barrier discharge $\mathrm{UV} / \mathrm{H}_{2} \mathrm{O}_{2}$. Detailed discussion on their advantages and disadvantages could be seen in the report of KFR (2011). In general, MP removal efficiency

Table 5

Environmental considerations of advanced treatment methods.

\begin{tabular}{|c|c|c|c|c|c|c|}
\hline & \multicolumn{2}{|c|}{ Activated carbon } & \multicolumn{2}{|l|}{ Advanced oxidation } & \multirow{2}{*}{$\begin{array}{l}\text { Membrane processes } \\
(\mathrm{NF} / \mathrm{RO})\end{array}$} & \multirow{2}{*}{$\begin{array}{l}\text { Membrane } \\
\text { bioreactors }\end{array}$} \\
\hline & PAC & GAC & $\mathrm{O}_{3}$ and $\mathrm{O}_{3}-\mathrm{AOPs}$ & $\mathrm{UV} / \mathrm{H}_{2} \mathrm{O}_{2}$ & & \\
\hline $\begin{array}{l}\text { Energy } \\
\text { consumption }\end{array}$ & $\begin{array}{l}\text { - Low ener- } \\
\text { gy }\end{array}$ & consumption & $\begin{array}{l}\text { Low energy } \\
\text { consumption, except } \\
\text { for onsite regeneration } \\
\text { of GAC (only feasible if } \\
\text { annual GAC consump- } \\
\text { tion } \geq 150,000 \mathrm{~kg} \text { ) }\end{array}$ & $\begin{array}{l}\text { - High energy } \\
\text { consumption } \\
+0.01-0.015 \mathrm{kWh} / \mathrm{m}^{3} \\
\mathrm{O}_{2} \\
+\mathrm{O}_{3} \text { production \& } \\
\text { control: } 12 \mathrm{kWh} / \mathrm{kg} \mathrm{O}_{3} \text {. } \\
85 \% \text { of energy is wasted } \\
\text { in form of heat. } \\
+ \text { treatment process: } \\
0.212 \mathrm{kWh} / \mathrm{m}^{3} \\
\text { wastewater }\end{array}$ & $\begin{array}{l}\text { - High energy } \\
\text { consumption } \\
+ \text { UV dose for MP removal } \\
\text { is } 5-6 \text { times higher than } \\
\text { being used for disinfection }\end{array}$ & $\begin{array}{l}\text { - High energy } \\
\text { consumption }\end{array}$ \\
\hline \multicolumn{7}{|c|}{$\begin{array}{l}\text { - Moderately } \\
\text { high energy } \\
\text { consumption } \\
\text { for aeration } \\
\text { \& pumping }\end{array}$} \\
\hline $\begin{array}{l}\text { Use and } \\
\text { handling of } \\
\text { chemicals }\end{array}$ & - None & - None & $\begin{array}{l}\text { Potential fire hazards } \\
\text { and toxicity risks of } \mathrm{O}_{3} \\
\text { handling }\end{array}$ & - None & $\begin{array}{l}\text { Chemicals for membrane } \\
\text { cleaning }\end{array}$ & $\begin{array}{l}\text { - Chemicals for } \\
\text { membrane } \\
\text { cleaning }\end{array}$ \\
\hline By-products & - None & - None & $\begin{array}{l}\text { Formation of by-- } \\
\text { products such as } \\
\text { NDMA, bromates, } \\
\text { carboxyl, aldehydes, } \\
\text { ketones } \\
\text { May be toxic }\end{array}$ & $\begin{array}{l}\text { - Formation of by-- } \\
\text { products such as } \\
\text { ketones, carboxyl, } \\
\text { aldehydes, etc. } \\
\text { - Residual } \mathrm{H}_{2} \mathrm{O}_{2} \text { must } \\
\text { be treated }\end{array}$ & $\begin{array}{l}\text { - Formation of by-- } \\
\text { products such as NDMA } \\
\text { due to using chloramine } \\
\text { to clean membranes }\end{array}$ & - None \\
\hline $\begin{array}{l}\text { Waste } \\
\text { production } \\
\text { and disposal }\end{array}$ & $\begin{array}{l}\text { - Increase } \\
5-10 \% \text { of } \\
\text { sludge vol- } \\
\text { ume } \\
\text { - Disposal of } \\
\text { used PAC }\end{array}$ & $\begin{array}{l}\text { - Spent GAC required regeneration } \\
\text { or replacement }\end{array}$ & - None & - None & $\begin{array}{l}\text { - Treatment of } \\
\text { concentrate }\end{array}$ & $\begin{array}{l}\text { - Treatment of } \\
\text { concentrate and } \\
\text { sludge contain- } \\
\text { ing MPs }\end{array}$ \\
\hline
\end{tabular}


Table 6

Technological considerations of advanced treatment methods.

\begin{tabular}{|c|c|c|c|c|c|c|}
\hline \multirow[t]{2}{*}{ Methods } & \multicolumn{2}{|l|}{ Activated carbon } & \multicolumn{2}{|l|}{ Advanced oxidation } & \multirow{2}{*}{$\begin{array}{l}\text { Membrane } \\
\text { processes }\end{array}$} & \multirow[t]{2}{*}{ Membrane bioreactors } \\
\hline & PAC & GAC & $\mathrm{O}_{3}$ and $\mathrm{O}_{3}-\mathrm{AOPs}$ & $\mathrm{UV} / \mathrm{H}_{2} \mathrm{O}_{2}$ & & \\
\hline $\begin{array}{l}\text { Ease of } \\
\text { construction }\end{array}$ & $\begin{array}{l}\text { Easy to } \\
\text { construct, in- } \\
\text { stall and } \\
\text { incorporate }\end{array}$ & $\begin{array}{l}\text { Easy to construct, install } \\
\text { and incorporate }\end{array}$ & $\begin{array}{l}\text { - } \mathrm{O}_{3} \text { applications require compli- } \\
\text { cated apparatus \& efficient } \\
\text { contacting systems. }\end{array}$ & $\begin{array}{l}\text { - Special reactor de- } \\
\text { sign for UV radiation }\end{array}$ & $\begin{array}{l}\text { - Commercially } \\
\text { available }\end{array}$ & $\begin{array}{l}\text { - Commercially avail- } \\
\text { able in the form of } \\
\text { modules }\end{array}$ \\
\hline Flexibility & $\begin{array}{l}\text { - Highly flexible } \\
\text { - Canbe applied } \\
\text { as needed }\end{array}$ & - Flexible & $\begin{array}{l}\text { - Be affected greatly by influent } \\
\text { COD, BOD and SS }\end{array}$ & $\begin{array}{l}\text { Not applicable for } \\
\text { low UV-transmission } \\
\text { wastewater }\end{array}$ & $\begin{array}{l}\text { Be subject to } \\
\text { pre-treated } \\
\text { water quality }\end{array}$ & $\begin{array}{l}\text { - Can accommodate a } \\
\text { wide range of influ- } \\
\text { ent fluctuation }\end{array}$ \\
\hline Reliability & $\begin{array}{l}\text { Lower efficiency } \\
\text { in the presence } \\
\text { ofDOC }\end{array}$ & $\begin{array}{l}\text { - Lower efficiency in the } \\
\text { presence of DOC }\end{array}$ & $\begin{array}{l}\text { Difficult to predict the forma- } \\
\text { tion of by-products } \\
\text { - Inefficient for wastewater with } \\
\text { high } \mathrm{O}_{3} \text { scavengers }\end{array}$ & $\begin{array}{l}\text { Difficult to predict } \\
\text { the formation of } \\
\text { by-products }\end{array}$ & $\begin{array}{l}\text { - High quality } \\
\text { effluent }\end{array}$ & - High quality effluent \\
\hline $\begin{array}{l}\text { Ease of } \\
\text { operation }\end{array}$ & $\begin{array}{l}\text { - Simple } \\
\text { operation }\end{array}$ & - Simple operation & $\begin{array}{l}\text { - Require professional proficient } \\
\text { - Ozone in off-gases from contact } \\
\text { chamber must be removed. } \\
\text { - Retain } \mathrm{O}_{3} \text { contact with MPs }\end{array}$ & $\begin{array}{l}\text { Require professional } \\
\text { proficient }\end{array}$ & $\begin{array}{l}\text { - Require profes- } \\
\text { sional profi- } \\
\text { cient } \\
\text { - Membrane } \\
\text { cleaning }\end{array}$ & $\begin{array}{l}\text { - Require professional } \\
\text { proficient } \\
\text { - Membrane cleaning }\end{array}$ \\
\hline $\begin{array}{l}\text { Maintenance } \\
\text { requirements }\end{array}$ & $\begin{array}{l}\text { - Separation of } \\
\text { PAC }\end{array}$ & $\begin{array}{l}\text { Replacement/regeneration } \\
\text { of adsorbents }\end{array}$ & $\begin{array}{l}\text { - } \mathrm{O}_{3} \text { is a very corrosive and reac- } \\
\text { tive substance } \rightarrow \text { require high-- } \\
\text { quality materials }\end{array}$ & $\begin{array}{l}\text { - UV lamp \& dosage of } \\
\mathrm{H}_{2} \mathrm{O}_{2}\end{array}$ & $\begin{array}{l}\text { Severe mem- } \\
\text { brane fouling }\end{array}$ & $\begin{array}{l}\text { - Severe membrane } \\
\text { fouling }\end{array}$ \\
\hline
\end{tabular}

between these methods in a similar operating condition decreases in the following order: medium pressure $\mathrm{UV} / \mathrm{H}_{2} \mathrm{O}_{2} \mathrm{~N}$ dielectric barrier discharge $\mathrm{UV} / \mathrm{H}_{2} \mathrm{O}_{2} \mathrm{~N}$ low pressure $\mathrm{UV} / \mathrm{H}_{2} \mathrm{O}_{2}$. Application of medium pressure $\mathrm{UV} / \mathrm{H}_{2} \mathrm{O}_{2}$ at the dosage of $450 \mathrm{~mJ} / \mathrm{cm}^{2}$ eliminated about $80 \%$ of MPs (KFR, 2011).

\subsubsection{Membrane processes}

Membrane fouling is the critical operational issue of membrane processes, especially RO. Membrane fouling types and causes are reviewed extensively in the literature (Guo et al., 2012). RO operation relies greatly on influent pre-treatment. In a survey of eight full-scale RO plants for water reclamation in Spain, Lazarova et al. (2008) found that poor pretreated water quality may decrease membrane lifetime by 60-75\%. The main fouling mechanism was organic fouling and biofilm formation on membrane surface (Lazarova et al., 2008). Polyamide is more sensitive to bio-fouling than other membrane materials. Therefore, operation of membrane processes demands professional proficient to (i) properly report system data (membrane cleaning/replacement schedule, system flow, $\mathrm{pH}$, pressure difference, turbidity and conductivity) and quality of feed, permeate and concentrate, (ii) analyse monitored data to commence repair or corrective maintenance, (iii) predict fouling types and time to clean or replace membrane to ensure a desired flux.

\subsubsection{Membrane bioreactors}

The membrane bioreactors have been applied widely in recent years, mostly in small-scale WWTPs. Although they can accommodate a wide range of influent fluctuation and attract interests from scientists, MBRs are still considered a risky and costly alternative for MP removal (Radjenovic et al., 2008). It requires highly skilled staff to operate. Additionally, membrane fouling in MBRs is very severe. The research carried out by C. Li et al. (2015) confirmed that some long-chain MPs such as carbamazepine can significantly increase the membrane fouling.

\subsubsection{Summary}

To sum up, main technological aspects from the selected treatment methods are presented in Table 6.

\subsection{Economic assessment}

It is very difficult to make a direct comparison between these alternatives in different countries, and sometimes even in the same country, because each treatment train is unique to its design criteria (WWTP's serving size, wastewater characteristics, targeted pollutants, removal requirements and specific site conditions) and operating conditions.

As presented in Sections 2.1 and 3.2, ozone and activated carbon adsorption are the preferential methods for MP removal in the pioneering European countries. Consequently, information on their financial appraisal is rather sufficient whereas economic assessment on membrane processes and MBRs is hardly available. Therefore, this section will discuss the cost analysis of these methods in detail. The framework for economic assessment of three common treatment methods (GAC, PAC/ sand filtration, $\mathrm{O}_{3} /$ sand filtration) is presented in Fig. 2.

\subsubsection{Activated carbon adsorption and ozonation}

In this section, a majority of raw data was taken from Mulder et al. (2015) and will be assessed against other studies (see Table 7).

3.5.1.1. Activated carbon adsorption. Despite activated carbon adsorption is simple to establish and operate, it bears a high capital investment and variable costs. The general design parameters for different studies are

\section{Table 7}

General design parameters for PAC + sand filter from experience of Germany, Switzerland and the Netherlands (data adapted from (Mulder et al., 2015)).

\begin{tabular}{|c|c|c|}
\hline PAC & Germany and Switzerland & Netherlands \\
\hline Dosage & $\begin{array}{l}0.7-1.4 \mathrm{~g} \mathrm{PAC} / \mathrm{g} \text { DOC } \\
10-20 \mathrm{mg} \mathrm{PAC} / \mathrm{L}\end{array}$ & $\begin{array}{l}1.1 \mathrm{~g} \mathrm{PAC} / \mathrm{g} \mathrm{DOC} \\
12 \mathrm{mg} \mathrm{PAC} / \mathrm{L}\end{array}$ \\
\hline Dosage coagulant & $4-6 \mathrm{mg} / \mathrm{L}$ & $5 \mathrm{mg} / \mathrm{L}$ \\
\hline Dosage polymer & $0.2-0.3 \mathrm{mg} 100 \%$ active $/ \mathrm{L}$ & $0.2 \mathrm{mg} 100 \%$ active $/ \mathrm{L}$ \\
\hline HRT & $30-40 \mathrm{~min}$ & $35 \mathrm{~min}$ \\
\hline Surface load settler & $2.0 \mathrm{~m} / \mathrm{h}$ & $2.0 \mathrm{~m} / \mathrm{h}$ \\
\hline PAC recycle rate & $0.5-1.0$ & 0.8 \\
\hline Power consumption & $45 \mathrm{~W} / \mathrm{m}^{3}$ treated water & $45 \mathrm{~W} / \mathrm{m}^{3}$ \\
\hline \multicolumn{3}{|c|}{ Sand filtration after $P A C$} \\
\hline Upflow velocity & $12 \mathrm{~m} / \mathrm{h}$ & \\
\hline Backwash water & $5-10 \%$ of influent & \\
\hline Power consumption & $15 \mathrm{~W} / \mathrm{m}^{3}$ treated water & $15 \mathrm{~W} / \mathrm{m}^{3}$ \\
\hline GAC & Germany and Switzerland & Netherlands \\
\hline $\begin{array}{l}\text { Empty bed contact } \\
\text { time }\end{array}$ & $20-40 \mathrm{~min}$ & $30 \mathrm{~min}$ \\
\hline Upflow velocity & $6-10 \mathrm{~m} / \mathrm{h}$ & $8 \mathrm{~m} / \mathrm{h}$ \\
\hline Replacement rate & $\begin{array}{l}\text { After } 7000-20,000 \text { bed } \\
\text { volumes } \\
\text { (every } 4 \text { months- } 1 \text { year) }\end{array}$ & $\begin{array}{l}8800 \text { bed volumes ( } 6 \\
\text { months) }\end{array}$ \\
\hline Power consumption & $40 \mathrm{~W} / \mathrm{m}^{3}$ treated water & $40 \mathrm{~W} / \mathrm{m}^{3}$ \\
\hline Backwash water & $5-15 \%$ of influent & $10 \%$ \\
\hline SS & & $\leq 10 \mathrm{mg} / \mathrm{L}$ \\
\hline
\end{tabular}


Table 8

General design parameters for ozone/sand filter from experience of Germany, Switzerland and the Netherlands (data adapted from (Mulder et al., 2015)).

\begin{tabular}{|c|c|c|}
\hline & Germany and Switzerland & Netherlands \\
\hline Dosage & $\begin{array}{l}0.6-0.9 \mathrm{~g} \mathrm{O}_{3} / \mathrm{g} \mathrm{DOC} \\
4-14 \mathrm{mg} \mathrm{O}_{3} / \mathrm{L} \\
\text { (based on DOC }=7-15 \mathrm{mg} / \mathrm{L} \text { ) }\end{array}$ & $\begin{array}{l}0.7 \mathrm{~g} \mathrm{O}_{3} / \mathrm{g} \mathrm{DOC}^{3} \\
7.7 \mathrm{mg} \mathrm{O}^{3} / \mathrm{L}\end{array}$ \\
\hline HRT & $\begin{array}{l}15-30 \mathrm{~min} \\
\text { (reactor } 10-25 \mathrm{~min}+\text { removing } \\
\text { remaining } \mathrm{O}_{3} 5 \mathrm{~min} \text { ) }\end{array}$ & $25 \mathrm{~min}$ \\
\hline $\begin{array}{l}\text { Power consumption for } \mathrm{O}_{3} \\
\text { production }\end{array}$ & $10 \mathrm{kWh} / \mathrm{kg} \mathrm{O}$ & $10 \mathrm{kWh} / \mathrm{kg} \mathrm{O}$ \\
\hline $\begin{array}{l}\text { Power consumption for } \\
\text { water treatment }\end{array}$ & $45 \mathrm{~W} / \mathrm{m}^{3}$ & $45 \mathrm{~W} / \mathrm{m}^{3}$ \\
\hline \multicolumn{3}{|l|}{ Sand filtration after $\mathrm{O}_{3}$} \\
\hline Upflow velocity & $12 \mathrm{~m} / \mathrm{h}$ & $12 \mathrm{~m} / \mathrm{h}$ \\
\hline Backwash water & $5-10 \%$ of influent & $5 \%$ \\
\hline Power consumption & $15 \mathrm{~W} / \mathrm{m}^{3}$ treated water & $\begin{array}{l}15 \mathrm{~W} / \mathrm{m}^{3} \\
\text { treated water }\end{array}$ \\
\hline
\end{tabular}

presented as follows, based on the frequently observed WWTP effluent's DOC $=7-15 \mathrm{mg} / \mathrm{L}$. The increase in DOC concentration will reduce the performance of PAC/GAC adsorption and require higher dosages, which in turn increase operational costs.

The onsite regeneration of GAC is so costly that it is only feasible when the carbon capacity is larger than $150,000 \mathrm{~kg}$ per year (Lee et al., 2009).

3.5.1.2. Ozonation. The operation and maintenance costs for ozone treatment consist of energy, chemicals and supplies, equipment repairs, and personnel requirements. The investment cost of upgrading WWTPs with an add-on ozonation step in Switzerland increases by $10-20 \%$ for large-scale ones and $20-50 \%$ for small- and medium-scale ones while energy consumption raised by 5-30\% (Eggen et al., 2014). The general design parameters for ozone treatment are presented in Table 8.

3.5.1.3. Comparison between activated carbon adsorption and ozonation. The MP removal costs for one $\mathrm{m}^{3}$ of WWTP effluent of three alternatives (i) Ozone + sand filtration, (ii) PAC + sand filtration and (iii) GAC filtration are illustrated in Fig. 3. These alternatives are proposed for three categories of WWTPs, including (1) small-scale WWTPs serving 20,000 people with the dry-weather wastewater flow-rate $200 \mathrm{~m}^{3} / \mathrm{h}$, (2) medium-scale WWTPs for 100,000 people with the design flowrate $1050 \mathrm{~m}^{3} / \mathrm{h}$, and (3) large-scale WWTPs for 300,000 people with $3100 \mathrm{~m}^{3} / \mathrm{h}$. The MP post-treatment cost with ozone and PAC technologies ranged $€ 0.22-0.26 / \mathrm{m}^{3}$ (equivalent to $0.24-0.31 \mathrm{USD} / \mathrm{m}^{3}$ ) for small plants and significantly reduced to $€ 0.16-0.18 / \mathrm{m}^{3}(0.17-0.20$ $\mathrm{USD} / \mathrm{m}^{3}$ ) for large-scale plants (Table 9 ). GAC is the most expensive option and its treatment cost has only been reduce slightly from smallscale to large-scale plants. GAC's variable costs were extremely high, which took account $65-75 \%$ of its annual expenditure. Interestingly, its capital investment is only a half of those of ozone and PAC treatments.

In this study, ozone with sand filtration was the most economical option in all scales of treatment; yet PAC with sand filtration is a comparable option for large-scale plants. This finding was in harmony with the

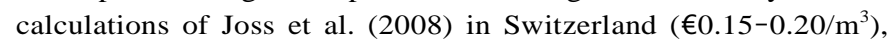
Roccaro et al. (2013) in Italia $\left(€ 0.30-0.40 / \mathrm{m}^{3}\right)$ and Wahlberg et al. (2010) in Sweden $\left(€ 0.15-0.30 / \mathrm{m}^{3}\right)$. The combination between PAC

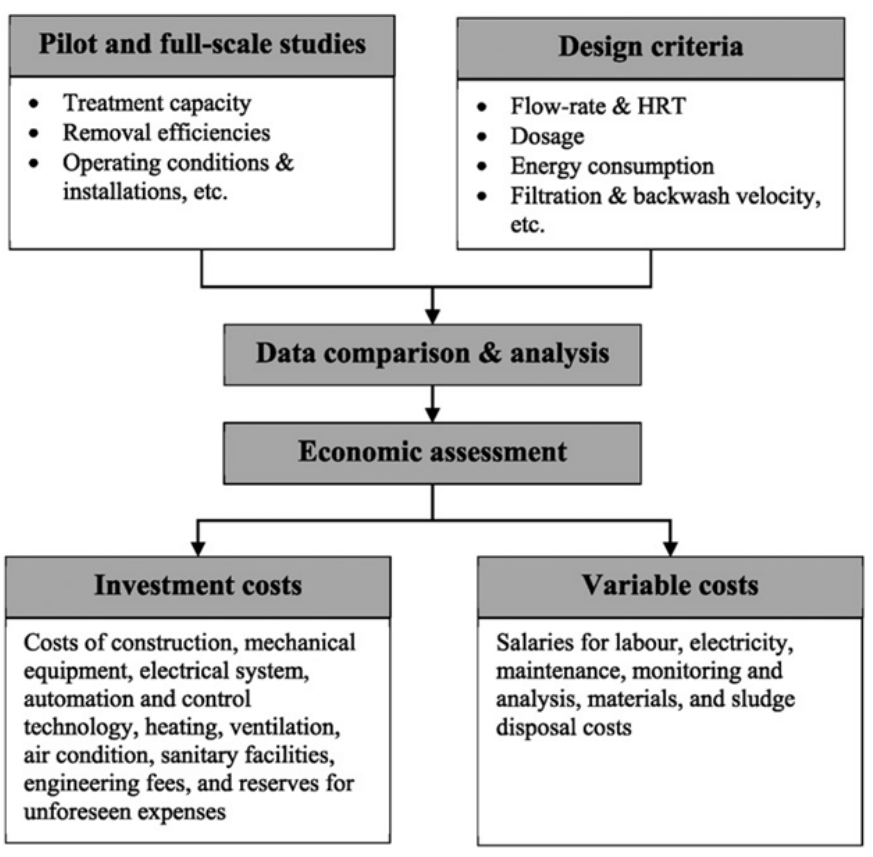

Fig. 2. Economic assessment framework for GAC, PAC and ozone treatment with available information from Germany, Switzerland and the Netherlands.

and UF can significantly increase the operational cost (Margot et al., 2013).

\subsubsection{Comparison with other treatment processes}

Due to the lack of information on economic evaluation from the other treatment processes, Fig. 4 was provided as a rough estimation for the relativity between MP treatment methods. It presented the results from Stockholm Water Company's four-year project "Pharmaceuticals - Presence and effects in the aquatic environment, preventive measures and possible treatment methods", reported by Wahlberg et al. (2010). 78 out of 90 studied pharmaceuticals were found in WWTPs' influents, such as hydrochlorothiazide, amiloride, propranolol, metoprolol, atorvastatin, amlodipine, to name just a few. In this figure, the residues left after treatment (\%) were calculated based on the MP reduction efficiencies of those selected treatment methods.

From Fig. 4, the most economical advanced treatment options may be ozonation and activated carbon adsorption. They also can minimize the residues of MPs wastewater to the lowest level. The cost will fluctuate greatly depending on the scale of treatment, treatment targets and so on. It could be a large burden to the plants itself, in case of lacking funding from the government.

\subsection{Social aspects}

The last aspect to be consider is public acceptance of different treatment methods in MP control. So far, no paper has dealt with this issue, which is an interesting domain for future research. However, learning from the experience of Switzerland, people are willing to pay up to $\$ 37 \pm 8$ on the top of their water bill to reduce the risk of MPs (Logar et al., 2014). The higher perception they have towards environmental issues, the higher value they agree to contribute to solve the

Table 9

Comparison of calculated costs in Germany, Switzerland and the Netherlands for MP removal including post treatment in a sand filter.

\begin{tabular}{llll}
\hline & Germany & Switzerland & Netherlands \\
\hline Small-scale WWTPs & $€ 0.21 \pm 0.08$ & $€ 0.15-0.30$ & $€ 0.22-€ 0.26 \pm 0.05$ \\
Medium-scale WWTPs & $€ 0.19 \pm 0.08$ & & $€ 0.18-€ 0.20 \pm 0.05$ \\
Large-scale WWTPs & $€ 0.14 \pm 0.08$ & $€ 0.05-0.11$ & $€ 0.16-€ 0.18 \pm 0.05$ \\
\hline
\end{tabular}




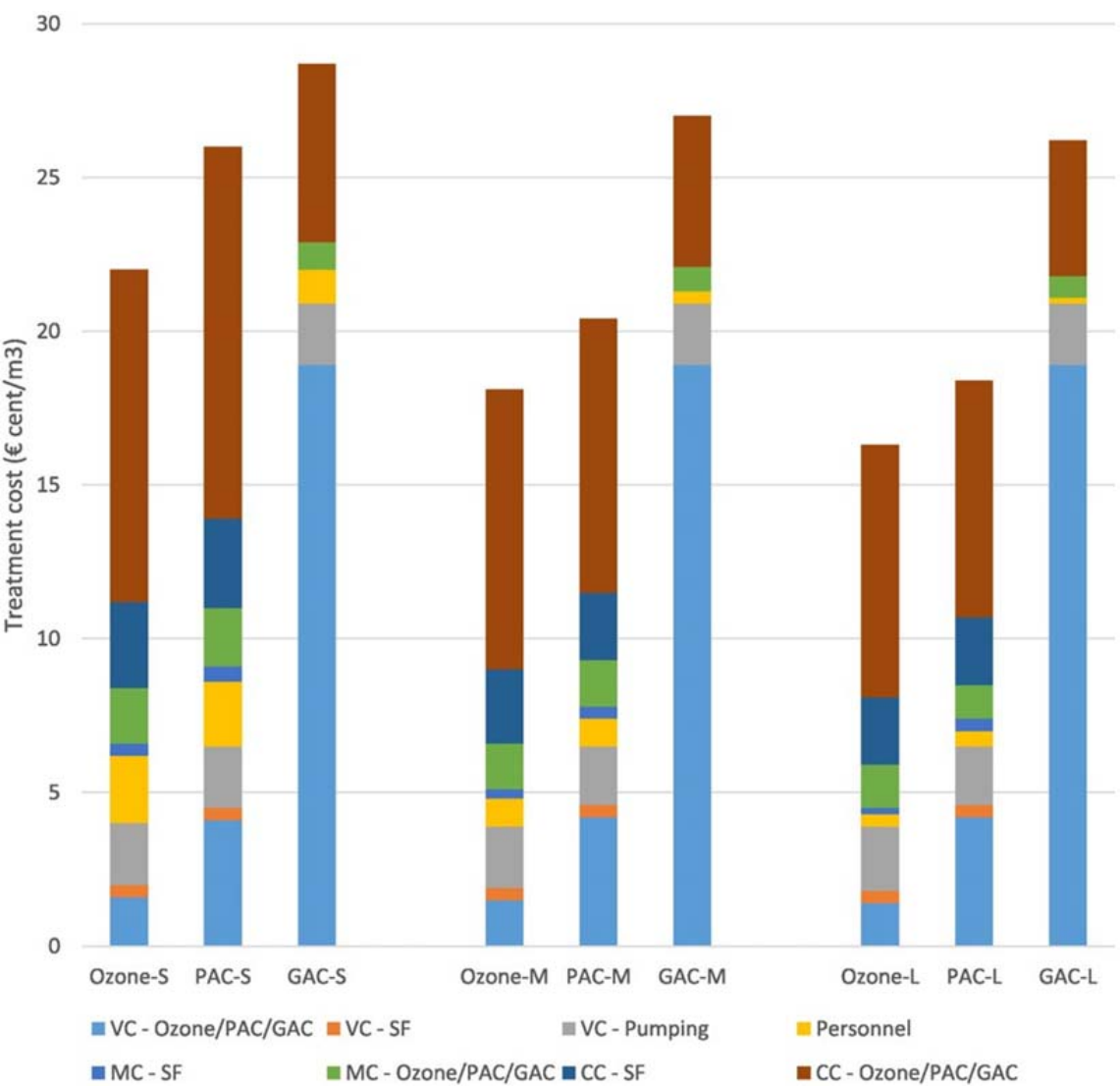

Fig. 3. Comparison of annual costs per $\mathrm{m}^{3}$ of WWTP effluent in the Netherlands for three advanced MP treatment options: (1) Ozone + sand filtration, (2) PAC + sand filtration and (3) GAC filtration (data adapted from (Mulder et al., 2015)). Notes: • SF: sand filtration • VC: variable cost, CC: capital cost, MC: maintenance cost • S: small-scale (WWTPs serving 20,000 people), M: medium-scale (100,000 people), L: large-scale (300,000 people) • GAC treatment option does not include sand filtration (VC-SF, CC-SF and MC-SF) • Value presented in Euro cent, by the time of March 2015

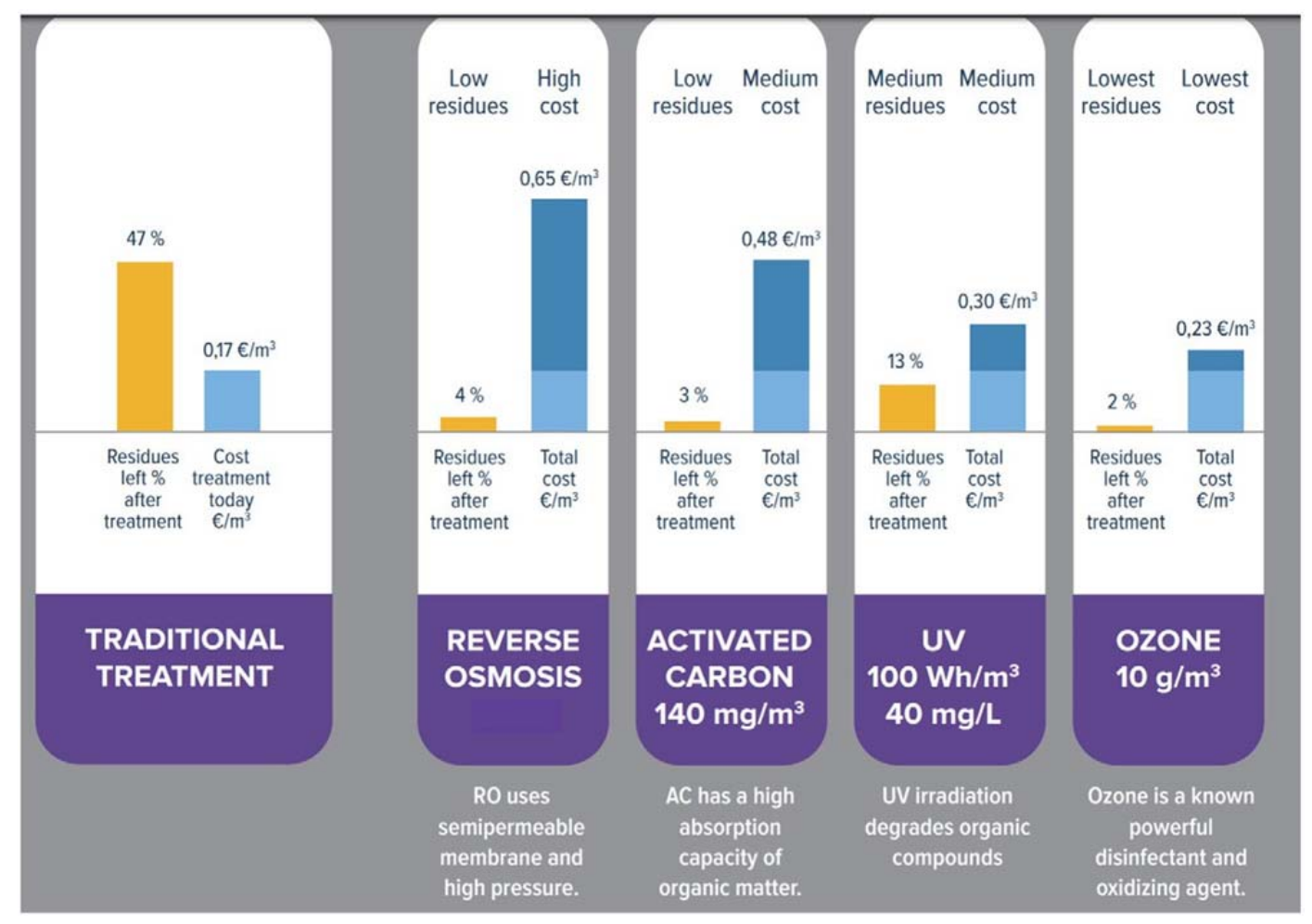


environmental problems. Public media are a very useful domain to help to educate people about the risk of MP contamination on water resources and benefits from upgrading the WWTPs to reduce its risk, especially in the regions with the increased trend of water reuse.

\section{Conclusions}

Based on the multicriteria analysis of MP treatment methods using full-scale and pilot-scale studies, several conclusions are summarised as follow:

- MPs are posing a new threat to the water environment, which urges scientist community and legislative bodies to work together and establish the regulations to control MPs.

- Switzerland is the first country that tackles the MP discharge at the national level. Their policy was officially approved in regulation system and received positive supports from all relevant stakeholders.

- Due to the diversity in nature of MPs, one single treatment technology is incapable to control all types of MPs. Each treatment method still has its drawbacks which need to be resolved. As a result, the combination of different advanced methods is an interesting research topic.

- From the specific case studies, ozonation and activated carbon adsorption have a longer history as advanced technologies to upgrade traditional WWTPs as they (i) have acceptable removal performance, (ii) are technically feasible, (iii) are sufficiently cost-effective. Nevertheless, the other methods are also promising and worth to be studied thoroughly.

After reviewing different methods for MP removal, some relevant topics are recommended for future research such as combination of various advanced methods for MP removal, economic analysis of membrane and MBR processes for MP treatment and social acceptance of each treatment methods.

\section{References}

Abejon, R., De Cazes, M., Belleville, M.P., Sanchez-Marcano, J., 2015. Large-scale enzymatic membrane reactors for tetracycline degradation in WWTP effluents. Water Res. 73, 118-131.

Altmann, J., Ruhl, A.S., Zietzschmann, F., Jekel, M., 2014. Direct comparison of ozonation and adsorption onto powdered activated carbon for micropollutant removal in advanced wastewater treatment. Water Res. 55, 185-193.

Audenaert, W.T.M., Chys, M., Auvinen, H., Dumoulin, A., Rousseau, D., Hulle, S.W.H.V., 2014. (Future) regulation of trace organic compounds in WWTP effluents as a driver of advanced wastewater treatment. Ozone News 42 (6), 17-23.

Beier, S., Cramer, C., Köster, S., Mauer, C., Palmowski, L., Schröder, H.F., Pinnekamp, J., 2011. Full scale membrane bioreactor treatment of hospital wastewater as forerunner for hot-spot wastewater treatment solutions in high density urban areas. Water Sci. Technol. 63 (1), 66-71.

Bernhard, M., Müller, J., Knepper, T.P., 2006. Biodegradation of persistent polar pollutants in wastewater: comparison of an optimised lab-scale membrane bioreactor and activated sludge treatment. Water Res. 40 (18), 3419-3428.

Blackbeard, J., Lloyd, J., Magyar, M., Mieog, J., Linden, K.G., Lester, Y., 2016. Demonstrating organic contaminant removal in an ozone-based water reuse process at full scale. Environ. Sci. Water Res. Technol. 2 (1), 213-222.

Cazes, M.d., Abejon, R., Belleville, M.-P., Sanchez-Marcano, J., 2014. Membrane bioprocesses for pharmaceutical micropollutant removal from waters. Membranes 4, 692-729.

Chedeville, O., Barrot, Y., Versaveau, F., Pineau, A., Cagnon, B., 2014. Endocrine disrupter removal by ozone/activated carbon coupling in continuous flow at pilot scale. J. Environ. Eng. 141 (5), 04014089.

Chelme-Ayala, P., Smith, D.W., El-Din, M.G., 2009. Membrane concentrate management options: a comprehensive critical review. Can. J. Civ. Eng. 36 (6), 1107-1119.

Creek, D., Davidson, J., 2000. Chapter 4 'granular activated carbon'. In: Meline, G. (Ed.), Treatment Technologies for Removal of Methyl Tertiary Butyl Ether (MTBE) from Drinking Water, second ed. Center for Groundwater Restoration and Protection - National Water Research Institute, California.

de Cazes, M., Belleville, M.P., Petit, E., Llorca, M., Rodríguez-Mozaz, S., de Gunzburg, J., Barceló, D., Sanchez-Marcano, J., 2014. Design and optimization of an enzymatic membrane reactor for tetracycline degradation. Catal. Today 236, 146-152.

de la Torre, T., Alonso, E., Santos, J.L., Rodriguez, C., Gomez, M.A., Malfeito, J.J., 2015. Trace organics removal using three membrane bioreactor configurations: MBR, IFAS-MBR and MBMBR. Water Sci. Technol. 71 (5), 761-768.
De Wever, H., Weiss, S., Reemtsma, T., Vereecken, J., Müller, J., Knepper, T., Hernando, M.D., 2007. Comparison of sulfonated and other micropollutants removal in membrane bioreactor and conventional wastewater treatment. Water Res. 41 (4), 935945.

Degremont, 2013. What are Micropollutants. Degremont (Available at: http://www. degremont.com/en/news/special-topics/what-are-micropollutants/, Accessed: 10// 08/20152015).

Derco, J., Dudáś, J., Valičková, M., Šimovičová, K., Kecskés, J., 2015. Removal of micropollutants by ozone based processes. Chem. Eng. Process. Process Intensif.

Eggen, R.I.L., Hollender, J., Joss, A., Schärer, M., Stamm, C., 2014. Reducing the discharge of micropollutants in the aquatic environment: the benefits of upgrading wastewater treatment plants. Environ. Sci. Technol. 48 (14), 7683-7689.

Environmental Technology Initiative, 2016. Fact sheet - ozone disinfection. (Available at:) http://www.nesc.wvu.edu/pdf/WW/publications/eti/Ozone_Dis_tech.pdf (Accessed: 27/02/2016).

Farré, M. J. and Knight, N. (2012) Assessment of regulated and emerging disinfection byproducts in South East Queensland drinking water - Urban Water Security Research Alliance Technical Report No. 90, Queensland (Australia): UQ and GU.

Grover, D.P., Zhou, J.L., Frickers, P.E., Readman, J.W., 2011. Improved removal of estrogenic and pharmaceutical compounds in sewage effluent by full scale granular activated carbon: impact on receiving river water. J. Hazard. Mater. 185 (2-3), 1005-1011.

Guo, W., Ngo, H.-H., Li, J., 2012. A mini-review on membrane fouling. Bioresour. Technol. $122,27-34$.

Hai, F.I., Tessmer, K., Nguyen, L.N., Kang, J., Price, W.E., Nghiem, L.D., 2011. Removal of micropollutants by membrane bioreactor under temperature variation. J. Membr. Sci. $383(1-2), 144-151$.

Hai, F.I., Nghiem, L.D., Khan, S.J., Price, W.E., Yamamoto, K., 2014. Chapter 6 - wastewater reuse: removal of emerging trace organic contaminants. In: Hai, F.I., Yamamoto, K., Lee, C.-H. (Eds.), Membrane Biological Reactors: Theory, Modeling, Design, Management and Applications to Wastewater Reuse. IWA Publishing, London.

Hollender, J., Zimmermann, S.G., Koepke, S., Krauss, M., McArdell, C.S., Ort, C., Singer, H., von Gunten, U., Siegrist, H., 2009. Elimination of organic micropollutants in a municipal wastewater treatment plant upgraded with a full-scale post-ozonation followed by sand filtration. Environ. Sci. Technol. 43 (20), 7862-7869.

James, C.P., Germain, E., Judd, S., 2014. Micropollutant removal by advanced oxidation of microfiltered secondary effluent for water reuse. Sep. Purif. Technol. $127,77-83$.

Johnson, A.C., Sumpter, J.P., 2015. Improving the quality of wastewater to tackle trace organic contaminants: think before you act! Environ. Sci. Technol. 49 (7), 3999-4000.

Jones, O.A.H., Green, P.G., Voulvoulis, N., Lester, J.N., 2007. Questioning the excessive use of advanced treatment to remove organic micropollutants from wastewater. Environ. Sci. Technol. 41 (14), 5085-5089.

Joss, A., Siegrist, H., Ternes, T.A., 2008. Are we about to upgrade wastewater treatment for removing organic micropollutants? Water Sci. Technol. 57 (2), 251-255.

Kennedy, A.M., Reinert, A.M., Knappe, D.R., Ferrer, I., Summers, R.S., 2015. Full- and pilotscale GAC adsorption of organic micropollutants. Water Res. 68, 238-248.

KFR, 2011. New concepts of $U V / \mathrm{H}_{2} \mathrm{O}_{2}$ oxidation. Water Research Foundation, Nieuwegein.

Kruithof, J.C., Kamp, P.C., Martijn, B.J., 2007. UV/H2O2 treatment: a practical solution for organic contaminant control and primary disinfection. Ozone Sci. Eng. 29 (4), 273-280.

Lazarova, V., Gallego, S., Molina, V.G., Rougé, A.P., 2008. Problems of operation and main reasons for failure of membranes in tertiary treatment systems. Water Sci. Technol. 57 (11), 1777-1784.

Lee, C.E., Howe, J.J., Thomson, B.M., 2009. State of Knowledge of Pharmaceutical, Personal Care Product, and Endocrine Disrupting Compound Removal during Municipal Wastewater Treatment. The University of New Mexico, Mexico.

Lenntech, 2016. Ozone decomposition. (Available at:) http://www.lenntech.com/library/ ozone/decomposition/ozone-decomposition.htm (Accessed: 17/02/2016).

Li, C., Cabassud, C., Reboul, B., Guigui, C., 2015a. Effects of pharmaceutical micropollutants on the membrane fouling of a submerged MBR treating municipal wastewater: case of continuous pollution by carbamazepine. Water Res. 69, 183-194.

Li, X., Shi, H., Li, K., Zhang, L., 2015b. Combined process of biofiltration and ozone oxidation as an advanced treatment process for wastewater reuse. Front. Environ. Sci. Eng.

Logar, I., Brouwer, R., Maurer, M., Ort, C., 2014. Cost-benefit analysis of the Swiss National Policy on reducing micropollutants in treated wastewater. Environ. Sci. Technol. 48 (21), 12500-12508.

Lowenberg, J., Zenker, A., Baggenstos, M., Koch, G., Kazner, C., Wintgens, T., 2014. Comparison of two PAC/UF processes for the removal of micropollutants from wastewater treatment plant effluent: process performance and removal efficiency. Water Res. 56, 26-36.

Luo, Y., Guo, W., Ngo, H.H., Nghiem, L.D., Hai, F.I., Zhang, J., Liang, S., Wang, X.C., 2014. A review on the occurrence of micropollutants in the aquatic environment and their fate and removal during wastewater treatment. Sci. Total Environ. 473-474, 619-641.

Mailler, R., Gasperi, J., Coquet, Y., Deshayes, S., Zedek, S., Cren-Olive, C., Cartiser, N., Eudes, V., Bressy, A., Caupos, E., Moilleron, R., Chebbo, G., Rocher, V., 2015. Study of a large scale powdered activated carbon pilot: removals of a wide range of emerging and priority micropollutants from wastewater treatment plant effluents. Water Res. 72, 315330.

Margot, J., Kienle, C., Magnet, A., Weil, M., Rossi, L., de Alencastro, L.F., Abegglen, C., Thonney, D., Chèvre, N., Schärer, M., Barry, D.A., 2013. Treatment of micropollutants in municipal wastewater: ozone or powdered activated carbon? Sci. Total Environ. 461-462, 480-498.

Meinel, F., Ruhl, A.S., Sperlich, A., Zietzschmann, F., Jekel, M., 2014. Pilot-scale investigation of micropollutant removal with granular and powdered activated carbon. Water Air Soil Pollut. 226 (1).

Metz, F., Ingold, K., 2014. Sustainable wastewater management: is it possible to regulate micropollution in the future by learning from the past? A policy analysis. Sustainability 6 (4), 1992. 
Miralles-Cuevas, S., Oller, I., Sanchez Perez, J.A., Malato, S., 2014. Removal of pharmaceuticals from MWTP effluent by nanofiltration and solar photo-Fenton using two different iron complexes at neutral pH. Water Res. 64, 23-31.

Mousaab, A., Claire, C., Magali, C., Christophe, D., 2015. Upgrading the performances of ultrafiltration membrane system coupled with activated sludge reactor by addition of biofilm supports for the treatment of hospital effluents. Chem. Eng. J. 262, 456-463.

Mulder, M., Antakyali, D., Ante, S., 2015. Costs of Removal of Micropollutants from Effluents of Municipal Wastewater Treatment Plants - General Cost Estimates for the Netherlands based on Implemented Full Scale Post Treatments of Effluents of Wastewater Treatment Plants in Germany and Switzerland. STOWA and Waterboard the Dommel, The Netherlands.

Nam, S.W., Choi, D.J., Kim, S.K., Her, N., Zoh, K.D., 2014. Adsorption characteristics of selected hydrophilic and hydrophobic micropollutants in water using activated carbon. J. Hazard. Mater. 270, 144-152.

NRMMC, Environment Protection and Heritage Council, National Health and Medica Research Council \& Natural Resource Management Ministerial Council, 2008a. Australian Guidelines for Water Recycling: Augmentation of Drinking Water Supplies. Biotext Pty Ltd., Canberra.

NRMMC, 2015. Australian Drinking Water Guidelines (2011) - updated 2015. Nationa Health and Medical Research Council, National Resource Management Ministerial Council, Commonwealth of Australia, Canberra.

Radjenovic, J., Matosic, M., Mijatovic, I., Petrovic, M., Barceló, D., 2008. Membrane bioreactor (MBR) as an advanced wastewater treatment technology. Handb. Environ. Chem. 5 (S2), 37-101.

Roccaro, P., Sgroi, M., Vagliasindi, F.G.A., 2013. Removal of xenobiotic compounds from wastewater for environment protection: treatment processes and costs. Chem. Eng. Trans. 32, 505-510.

Rodriguez-Mozaz, S., Ricart, M., Kock-Schulmeyer, M., Guasch, H., Bonnineau, C., Proia, L., de Alda, M.L., Sabater, S., Barcelo, D., 2015. Pharmaceuticals and pesticides in reclaimed water: efficiency assessment of a microfiltration-reverse osmosis (MFRO) pilot plant. J. Hazard. Mater. 282, 165-173.

Safe Work Australia, 2015. Hazardous Substances Information System - Ozone. Safe Work Australia, Australia (Available at: http://hsis.safeworkaustralia.gov.au/ ExposureStandards/Details? exposureStandardID=461).

Sarathy, S., Mohseni, M., 2008. An overview of UV-based advanced oxidation processes for drinking water treatment. IUAV News 8 (2), 16-27.

Siegrist, H., Joss, A., 2012. Review on the fate of organic micropollutants in wastewate treatment and water reuse with membranes. Water Sci. Technol. 66 (6), 1369-1376.
Stamm, C., Eggen, R.I.L., Hering, J.G., Hollender, J., Joss, A., Schärer, M., 2015. Micropollutant removal from wastewater: facts and decision-making despite uncertainty. Environ. Sci. Technol. 49 (11), 6374-6375.

Stasinakis, A.S., Gatidou, G., 2010. Chapter 1. Micropollutants and aquatic environment. In: Virkutyte, J., Varma, R.S., Jegatheesan, V. (Eds.), Treatment of Micropollutants in Water and Wastewater. IWA Publishing, London, UK, pp. 1-35.

Tadkaew, N., Hai, F.I., McDonald, J.A., Khan, S.J., Nghiem, L.D., 2011. Removal of trace organics by MBR treatment: the role of molecular properties. Water Res. 45 (8), 2439-2451.

The Council of EU, 1998. COUNCIL DIRECTIVE 98/83/EC of 3 November 1998 on the quality of water intended for human consumption. Official J. Eur. Communities L 330/32.

Thomaidi, V.S., Stasinakis, A.S., Borova, V.L., Thomaidis, N.S., 2015. Is there a risk for the aquatic environment due to the existence of emerging organic contaminants in treated domestic wastewater? Greece as a case-study. J. Hazard. Mater. 283, 740-747.

Trinh, T., van den Akker, B., Stuetz, R.M., Coleman, H.M., Le-Clech, P., Khan, S.J., 2012. Removal of trace organic chemical contaminants by a membrane bioreactor. Water Sci. Technol. 66 (9), 1856-1863.

USEPA, 2009. National Primary Drinking Water Regulations. EPA, USA.

Verlicchi, P., Al Aukidy, M., Zambello, E., 2015. What have we learned from worldwide experiences on the management and treatment of hospital effluent? - an overview and a discussion on perspectives. Sci. Total Environ. 514, 467-491.

Wahlberg, C., Björlenius, B., Paxéus, N., 2010. Läkemedelsrester i Stockholms vattenmiljö Förekomst, förebyggande åtgärder och rening av avloppsvatten (Pharmaceuticals Presence and effects in the aquatic environment, preventive measures and possible treatment methods). Stockholm Water Company, Stockholm.

WHO, 2011. Guidelines for drinking-water quality. fourth edition. WHO, Malta.

Yang, W., Zhou, H., Cicek, N., 2014. Treatment of organic micropollutants in water and wastewater by UV-based processes: a literature review. Crit. Rev. Environ. Sci. Technol. 44 (13), 1443-1476.

Yangali-Quintanilla, V., Maeng, S.K., Fujioka, T., Kennedy, M., Li, Z., Amy, G., 2011. Nanofiltration vs. reverse osmosis for the removal of emerging organic contaminants in water reuse. Desalin. Water Treat. 34 (1-3), 50-56.

Zwiener, C., 2006. Occurrence and analysis of pharmaceuticals and their transformation products in drinking water treatment. Anal. Bioanal. Chem. 387 (4), 1159-1162. 